\title{
Review Article \\ Phenomenology of Neutrino Mixing in Vacuum and Matter
}

\author{
A. Upadhyay and M. Batra \\ School of Physics and Material Science, Thapar University, Patiala, Punjab 147004, India \\ Correspondence should be addressed to M. Batra; mbatra310@gmail.com
}

Received 9 August 2012; Accepted 13 September 2012

Academic Editors: A. Belhaj, C. A. d. S. Pires, and A. Koshelev

Copyright (C) 2013 A. Upadhyay and M. Batra. This is an open access article distributed under the Creative Commons Attribution License, which permits unrestricted use, distribution, and reproduction in any medium, provided the original work is properly cited.

The current status and some perspectives of the phenomenology of massive neutrinos is reviewed. We start with the phenomenology of neutrino oscillations in vacuum and in matter. We summarize the results of neutrino experiments using solar, atmospheric. The fundamental theory of flavor changing neutrinos that has confirmed the neutrino oscillations and the various parameters affecting these oscillations have been discussed in detail. Specifically we will take the solar and atmospheric neutrino case. The oscillation plots will be discussed in detail, based on their behavior in vacuum and matter. Both normal and inverted mass hierarchy hypotheses are tested and both are consistent with observation. Finally the sensitivity of theta 13 over these probability oscillations has been analyzed and commented.

\section{Introduction}

Neutrinos are the most elusive in nature. They interact much less through coupling with $W^{+}, W^{-}$and, $Z^{0}$ boson. Several past and currently going neutrino experiments have not only resolved the mystery of solar and atmospheric neutrinos but also proved the massive nature of neutrinos. The flavor oscillation phenomenon leads to nonzero mass in the theories beyond a standard model in contradiction to the well-established standard model. The history of neutrino oscillation traces back to Gribov and Pontecorvo [1] but the credit for providing the evidence of neutrino oscillation goes to Cleveland et al. [2] for observing deficit in $v_{e}$ 's neutrino flux about $1 / 3$ rd as given by the standard solar model [3] through the chlorine Homestake experiment [2]. Several independent experiments like GALLEX [4] and SAGE [5] agreed with Cleveland et al. [2] by observing the neutrino flux more than half as expected from the solar model predictions. The mystery of solar neutrinos finally got resolved with the experiment Sudbury Neutrino Observatory [6] which proved that the deficit was due to flavor transitions. The experiment was different in the sense that it used a heavy water detector to detect ${ }^{8} \mathrm{~B}$ neutrinos via charged current interaction with electron only and neutral current interaction with total neutrino flux. Atmospheric neutrino anomaly is related to the electron-to-muon-neutrino ratio which reduces to half of the expected value and a possible explanation is the change of flavor from $v_{\mu} \Leftrightarrow v_{\tau}, v_{\mu} \Leftrightarrow v_{e}$. Solar and atmospheric neutrino oscillations, neutrino masses, and mixing parameters have been proved by various detectors with different sensitivity and accuracy. The recent journey of detectors in neutrino physics starts from Super-Kamiokande [7], KamLAND [8], T2 K [9], MINOS, [10] and finally end with detectors like SOUDAN [11] and OPERA [12]. The KamioKande [13] measured solar neutrinos to be half as per the SM whereas two experiments Gallex and Sage $[4,5]$ measured $56-60 \%$ of neutrino capture rate as predicted by the standard model. Then Super-Kamiokande I, II, and III [14-16] provided evidence for nonzero mass and also produced observation consistent with $\mu$-neutrinos changing into $\tau$-neutrinos. The latest searches at these experiments are more concerned with oscillation parameters, CP violation, and mass hierarchies. KAMLAND experiment was also able to investigate geographically produced antineutrinos $[15,17]$ and the best fitted values of $\Delta m_{21}^{2}=7.58+0.14$ (stat) ${ }_{-0.15}^{+0.15}$ (syst) $\times 10^{-5} \mathrm{eV}^{2}$ and $\tan \theta_{12}=0.56+{ }_{-0.07}^{0.10}$ (stat) ${ }_{-0.06}^{+0.10}$ (syst) for $\tan \theta_{12}<1$ as calculated using KAMLAND experiment. K2K $[16,18]$ was another long baseline experiment to study oscillation from $v_{\mu}$ to $v_{e}$ in the atmospheric region and confirmed the deficit of muon as observed in Super-Kamiokande. MINOS [17] at the Fermi National Laboratory studied muon oscillations 
produced from pion and kaon decay in the energy range of 1$10 \mathrm{GeV}$ and focused primarily on the measurement of $\Delta m_{23}^{2}$ with the precision better than $10 \%$.

The most recent parameter of our interest is $\theta_{13}$ because it has opened some of the most fundamental questions like CP violation. The nonzero value of $\theta_{13}$ may change the old picture of neutrino oscillation completely. Our main focus is the study of the flavor oscillations in the solar and atmospheric neutrinos. The detailed theory is presented with the aim that oscillation shows variations in vacuum and matter for the solar and atmospheric cases. The parameters affecting the neutrino oscillations are all included in our studies. Few experiments are analyzed in detail and their significance is mentioned. Special attention is given to the probability oscillation curve and its sensitivity to the recent bound on $\theta_{13}$.

\section{Theory}

Neutrino oscillations are periodic transitions between different flavor neutrinos in neutrino beams. The theory of neutrino oscillations has been studied by various pioneer scientists via different approaches including classical treatment by moving them to quantum mechanical treatment of wave packets [18] and then finally with quantum field theoretic treatment [19]. In this paper we present a detailed formalism for neutrino using the classical treatment only. The dependence of states on the time is given by the Schrodinger equation:

$$
i \frac{\partial|\Psi(t)\rangle}{\partial t}=H|\Psi(t)\rangle,
$$

where $H$ is the total Hamiltonian in flavor space and the general solution for (1) is

$$
|\Psi(t)\rangle=e^{-i H t}|\Psi(0)\rangle,
$$

where $|\Psi(0)\rangle$ is the state at the initial time $(t=0)$.

Flavor neutrino states and mass eigen states can be related through leptonic mixing matrix $U$ as

$$
\left|v_{l}\right\rangle=\sum_{i=0}^{3} U_{l i}^{*}\left|v_{i}\right\rangle .
$$

At time $(t=0)$,

$$
|\Psi(0)\rangle=\left|v_{i}\right\rangle .
$$

Application of the Hamiltonian operator will give the following:

$$
H\left|v_{i}\right\rangle=E_{i}\left|v_{i}\right\rangle
$$

where

$$
E_{i}=\sqrt{p_{i}^{2}+m_{i}^{2}} .
$$

The left-handed neutrino at the time $t \geq 0$ is written as

$$
\left|v_{l}\right\rangle_{t}=e^{-i H t}\left|v_{l}\right\rangle=\sum_{i=1}^{3} e^{-i E_{i} t} U_{l i}^{*}\left|v_{i}\right\rangle .
$$

Similarly,

$$
\left|\overline{v_{l}}\right\rangle_{t}=e^{-i H t}\left|\overline{v_{l}}\right\rangle=\sum_{i=1}^{3} e^{-i E_{i} t} U_{l i}\left|\overline{v_{i}}\right\rangle .
$$

Neutrino energies $E_{i}(i=1,2,3)$ are different; hence the flavor oscillation in neutrinos gives the amplitude of the transition $v_{l} \rightarrow v_{l^{\prime}}$ during the time $t$ and can be written as

$$
A\left(v_{l} \longrightarrow v_{l^{\prime}}\right)=\sum_{i=1}^{3} U_{i l^{\prime}} e^{-i E_{i} t} U_{l i}^{*}
$$

Analogously, the amplitude of the transition $\overline{v_{l}} \rightarrow \overline{v_{l^{\prime}}}$ during the time $t$ is given by

$$
A\left(\overline{v_{l}} \longrightarrow \overline{l_{l^{\prime}}}\right)=\sum_{i=1}^{3} U_{i l^{\prime}}^{*} e^{-i E_{i} t} U_{l i}
$$

Probability for the transition of neutrinos and antineutrinos will be

$$
\begin{aligned}
& P\left(v_{l} \longrightarrow v_{l^{\prime}}\right)=\left|\sum_{i=1}^{3} U_{i l^{\prime}} e^{-i E_{i} t} U_{l i}^{*}\right|^{2}, \\
& P\left(\overline{v_{l}} \longrightarrow \overline{v_{l^{\prime}}}\right)=\left|\sum_{i=1}^{3} U_{i l^{\prime}}^{*} e^{-i E_{i} t} U_{l i}\right|^{2} .
\end{aligned}
$$

From (11), we can find possible relations between probabilities:

$$
\begin{array}{ll}
\sum_{l} P\left(v_{l} \longrightarrow v_{l^{\prime}}\right)=1, & \sum_{l^{\prime}} P\left(\overline{v_{l}} \longrightarrow \overline{v_{l^{\prime}}}\right)=1, \\
\sum_{l^{\prime}} P\left(v_{l} \longrightarrow v_{l^{\prime}}\right)=1, & \sum_{l} P\left(\overline{v_{l}} \longrightarrow \overline{v_{l^{\prime}}}\right)=1 .
\end{array}
$$

A mixed neutrino state is characterized by their momentum $p$ with $p_{i}=p$ and mass $m_{i}$ with $m_{i}^{2} / p^{2} \ll 1$. And

$$
E_{i} \cong p+\frac{m_{i}^{2}}{2 p} .
$$

Hence the energy difference between two such states will be

$$
E_{i}-E_{j}=\frac{\Delta m_{j i}^{2}}{2 p},
$$

where $\Delta m_{j i}^{2}=m_{i}^{2}-m_{j}^{2}$.

As $E \approx p$, therefore

$$
E_{i}-E_{j}=\frac{\Delta m_{j i}^{2}}{2 E} .
$$

Here the assumption is made that neutrinos are having different energy in different mass eigenstates. Let us suppose the $t$ denotes the difference between production and detection 
time for the ultrarelativistic neutrinos and $L$ is the distance between source and the detector:

$$
\begin{gathered}
t \cong L, \\
\left(E_{i}-E_{j}\right) t=\frac{\Delta m_{j i}^{2}}{2 E} L, \\
P\left(v_{l} \longrightarrow v_{l^{\prime}}\right)=\left|\sum_{i=1}^{3} U_{l^{\prime} i} e^{-i\left(\Delta m_{j i}^{2} L / 2 E\right)} U_{l i}^{*}\right|^{2} .
\end{gathered}
$$

The unitary condition suggests that

$$
\sum_{i} U_{l^{\prime} i} U_{l i}^{*}=\delta_{l l^{\prime}}
$$

Hence

$$
P\left(v_{l} \longrightarrow v_{l^{\prime}}\right)=\left|\delta_{l l^{\prime}}+\sum_{i \neq j} U_{i l^{\prime}}\left(e^{-i\left(\Delta m_{j i}^{2} L / 2 E\right)}-1\right) U_{l i}^{*}\right|^{2} .
$$

Analogously, for the case of antineutrino,

$$
P\left(\overline{v_{l}} \longrightarrow \overline{\nu_{l^{\prime}}}\right)=\left|\delta_{l l^{\prime}}+\sum_{i \neq j} U_{i l^{\prime}}^{*}\left(e^{-i\left(\Delta m_{j i}^{2} L / 2 E\right)}-1\right) U_{l i}\right|^{2}
$$

The transition probability $v_{l} \rightarrow v_{l^{\prime}}$ is given as

$$
\begin{aligned}
P\left(v_{l} \longrightarrow v_{l^{\prime}}\right)= & \sum_{i, k} U_{l^{\prime} i} U_{l^{\prime} k}^{*} U_{l i}^{*} U_{l k} e^{-i\left(\Delta m_{j i}^{2} / 2 E\right) L} \\
= & \sum_{i}\left|U_{l^{\prime} i}\right|^{2}\left|U_{l i}\right|^{2} \\
& +2 \operatorname{Re} \sum_{i>k}\left(U_{l^{\prime} i} U_{l^{\prime} k}^{*} U_{l i}^{*} U_{l k} e^{-i\left(\Delta m_{j i}^{2} / 2 E\right) L}\right), \\
\sum_{i}\left|U_{l^{\prime} i}\right|^{2}\left|U_{l i}\right|^{2}= & \delta_{l l^{\prime}}-2 \operatorname{Re} \sum_{i>k}\left(U_{l^{\prime} i} U_{l k}^{*} U_{l i}^{*} U_{l k}\right) \\
= & \delta_{l l^{\prime}}-2 \operatorname{Re} \sum_{i>k}\left(U_{l^{\prime} i} U_{l^{\prime} k}^{*} U_{l i}^{*} U_{l k}\right) \\
& +2 \operatorname{Re} \sum_{i>k}\left(U_{l^{\prime} i} U_{l^{\prime} k}^{*} U_{l i}^{*} U_{l k} e^{-i\left(\Delta m_{j i}^{2} / 2 E\right) L}\right) \\
= & \delta_{l l^{\prime}}-2 \operatorname{Re} \sum_{i>k}\left(U_{l^{\prime} i} U_{l^{\prime} k}^{*} U_{l i}^{*} U_{l k}\right) \\
& \times\left(1-e^{-i\left(\Delta m_{k i}^{2} L / 2 E\right)}\right) .
\end{aligned}
$$

Finally for any complex $a$ and $b, \operatorname{Re}(a b)=\operatorname{Re}(a) \operatorname{Re}(b)-$ $\operatorname{Im}(a) \operatorname{Im}(b)$

$$
\begin{aligned}
P\left(v_{l} \longrightarrow v_{l^{\prime}}\right)= & \delta_{l l^{\prime}}-2 \operatorname{Re} \sum_{i>k}\left(U_{l^{\prime} i} U_{l^{\prime} i}^{*} U_{l i}^{*} U_{l k}\right) \\
& \times\left(1-\cos \frac{\Delta m_{k i}^{2} L}{2 E}\right) \\
& +2 \sum_{i>k} \operatorname{Im}\left(U_{l^{\prime} i} U_{l^{\prime} k}^{*} U_{l i}^{*} U_{l k}\right) \sin \frac{\Delta m_{k i}^{2} L}{2 E} .
\end{aligned}
$$

Similarly, for the antineutrino oscillation, probability becomes

$$
\begin{aligned}
P\left(v_{\bar{l}} \longrightarrow v_{l^{\prime}}\right)= & \delta_{l l^{\prime}}-2 \operatorname{Re} \sum_{i>k}\left(U_{l^{\prime} i} U_{l^{\prime} i}^{*} U_{l i}^{*} U_{l k}\right) \\
& \times\left(1-\cos \frac{\Delta m_{k i}^{2} L}{2 E}\right) \\
& -2 \sum_{i>k} \operatorname{Im}\left(U_{l^{\prime} i} U_{l^{\prime} k}^{*} U_{l i}^{*} U_{l k}\right) \sin \frac{\Delta m_{k i}^{2} L}{2 E} .
\end{aligned}
$$

\section{Two-Flavor Oscillation \\ Probability in Vacuum}

For two-flavor neutrino oscillations, the mixing matrix can be defined in terms of some rotating angle $\theta$ in 2 dimensions where $U$ is actually PMNS matrix; therefore

$$
\begin{gathered}
\left(\begin{array}{c}
v_{e} \\
v_{\mu}
\end{array}\right)=\left(\begin{array}{cc}
\cos \theta & \sin \theta \\
-\sin \theta & \cos \theta
\end{array}\right)\left(\begin{array}{l}
v_{1} \\
v_{2}
\end{array}\right), \\
\left|\nu_{e}(t=0)\right\rangle=\left|v_{e}\right\rangle=\cos \theta\left|v_{1}\right\rangle+\sin \theta\left|v_{2}\right\rangle, \\
\left|\nu_{\mu}(t=0)\right\rangle=\left|v_{\mu}\right\rangle=-\sin \theta\left|v_{1}\right\rangle+\cos \theta\left|v_{2}\right\rangle .
\end{gathered}
$$

The weak eigenstates are rotated by an angle $\theta$ with respect to the mass eigenstates $\left|v_{1}\right\rangle$ and $\left|v_{2}\right\rangle$ to allow mixing between $v_{\mu}$ and $v_{e}$. After some time $t$

$$
\begin{aligned}
\left|\nu_{\mu}(t=t)\right\rangle= & \left|\nu_{\mu}(t)\right\rangle=-\sin \theta\left|\nu_{1}\right\rangle e^{\left(-i E_{1} t / h\right)} \\
& +\cos \theta\left|\nu_{2}\right\rangle e^{-i E_{2} t / h} \\
= & -\sin \theta\left|\nu_{1}\right\rangle e^{-i\left(p+\left(m_{1}^{2} / 2 p\right)\right) t / h} \\
& +\cos \theta\left|\nu_{2}\right\rangle e^{-i\left(p+\left(m_{2}^{2} / 2 p\right)\right) t / h},
\end{aligned}
$$

where we have used

$$
\begin{gathered}
E_{1}=\left(p^{2}+m_{1}^{2}\right)^{1 / 2}, \quad E_{2}=\left(p^{2}+m_{2}^{2}\right)^{1 / 2}, \\
P\left(v_{e} \longrightarrow v_{\mu}\right)=\left|\left\langle v_{\mu} \mid v_{e}(t)\right\rangle\right|^{2}, \\
\left\langle v_{\mu}\right|=\cos \theta\left\langle v_{1}\right|+\sin \theta\left\langle v_{2}\right|, \\
P\left(v_{e} \longrightarrow v_{\mu}\right)=\left|\left\langle v_{\mu} \mid v_{e}(t)\right\rangle\right|^{2} \\
=e^{i z-i z} \sin ^{2} \theta \cos ^{2} \theta\left(1-e^{\left(i \Delta m^{2} / 2 p\right) x}\right) \\
\times\left(1-e^{\left(-i \Delta m^{2} / 2 p\right) x}\right) .
\end{gathered}
$$


For neutrinos to be relativistic, the substitution $p=E_{v}$ and $x=L$ can be made:

$$
\begin{gathered}
P\left(v_{e} \longrightarrow v_{\mu}\right)=\sin ^{2} \theta \cos ^{2} \theta\left(1-e^{\left(i \Delta m^{2} / 2 E_{v}\right) L}\right) \\
\times\left(1-e^{\left(-i \Delta m^{2} / 2 E_{v}\right) L}\right) . \\
P\left(v_{e} \longrightarrow v_{\mu}\right)(L, E)=\sin ^{2} 2 \theta \sin ^{2}\left(1.27 \Delta m^{2} \frac{L}{E_{v}}\right) .
\end{gathered}
$$

Here $\theta$ represents the mixing between two mass eigen states, $L$ is length of source from the detector also known as baseline, $E$ is the energy of neutrinos produced from the source. The functional dependence on $L / E$ is called a spectral dependence where $F(L / E)=L / E$. The mass squared difference values and the mixing angle are the significant quantities to be measured. Physicists just probe the different mass eigenvalues and predict the mixing at which it occurs. Mixing angle dependence of the transition probability is expressed by $(\sin 2 \theta)^{2}$. If we change from $\theta$ to $\pi / 2-\theta$, the mixing angle dependence remains as such which confirms with degeneracy of oscillation the probability for $\theta$ and $\pi / 2-\theta$. Two possibilities here correspond to two physically different mixings for two mass eigen states: if $\theta<\pi / 4$, the electron neutrino is composed more of $\nu_{1}$, and if $\theta>\pi / 4$, then muon neutrino is composed more of $v_{2}$. Moreover, transition to a different flavor is not possible if $\Delta m^{2} L / 2 E \ll 1$ which led us to a survival probability. Direct information about the mixing angle can be obtained from the average neutrino oscillation probability $\left\langle P\left(v_{\alpha} \rightarrow v_{\beta}\right)\right\rangle=(1 / 2)(\sin 2 \theta)^{2}$.

\section{Three-Flavor Probability Oscillations in Vacuum}

In case of three-flavor neutrino oscillations, the standard parameterization of mixing matrix can be achieved by using three vectors, and performing the Euler rotations introduces three mixing angles and one complex phase factor. For three flavor and three mass eigen states, it can be written as

$$
\left(\begin{array}{l}
v_{1} \\
v_{2} \\
v_{3}
\end{array}\right)=U\left(\begin{array}{l}
v_{e} \\
v_{\mu} \\
v_{\tau}
\end{array}\right) .
$$

Consider the unitary $3 \times 3$ mixing matrix for the Dirac neutrinos and introduce the standard parameters (three mixing angles and one phase) which characterize it:

$$
\begin{gathered}
|i\rangle \quad(i=1,2,3), \\
\langle i \mid k\rangle=\delta_{i k} .
\end{gathered}
$$

The first Euler rotation performed at the angle $\theta_{12}$ around the vector $|3\rangle$ produces new orthogonal and normalized vectors as

$$
\begin{gathered}
|1\rangle^{(1)}=c_{12}|1\rangle+s_{12}|2\rangle, \\
|2\rangle^{(1)}=-s_{12}|1\rangle+c_{12}|2\rangle, \\
|3\rangle^{(1)}=|3\rangle .
\end{gathered}
$$

Here $c_{12}=\cos \theta_{12}$ and $s_{12}=\sin \theta_{12}$;

$$
\begin{gathered}
|v\rangle^{(1)}=U^{(1)}|v\rangle \quad \text { such that } \\
|v\rangle^{(1)}=\left(\begin{array}{c}
\left|1^{(1)}\right\rangle \\
\left|2^{(1)}\right\rangle \\
\left|3^{(1)}\right\rangle
\end{array}\right), \quad|v\rangle=\left(\begin{array}{l}
|1\rangle \\
|2\rangle \\
|3\rangle
\end{array}\right), \\
U^{(1)}=\left(\begin{array}{ccc}
c_{12} & s_{12} & 0 \\
-s_{12} & c_{12} & 0 \\
0 & 0 & 1
\end{array}\right) .
\end{gathered}
$$

Second rotation at the angle $\theta_{13}$ around vector the $|2\rangle^{(1)}$ introduces the CP phase $\delta$ :

$$
\begin{gathered}
\left.|1\rangle^{(2)}=c_{13}|1\rangle^{(1)}\left|+s_{13} e^{-i \delta}\right| 3\right\rangle^{(1)}, \\
|2\rangle^{(2)}=|2\rangle^{(1)}, \\
|3\rangle^{(2)}=-s_{13} e^{-i \delta}|1\rangle^{(1)}+c_{13}|3\rangle^{(1)} .
\end{gathered}
$$

In the matrix $|v\rangle^{(2)}=U^{(2)}|v\rangle^{(1)}$, where $U^{(2)}=$ $\left(\begin{array}{cccc}c_{13} & 0 & s_{13} e^{-i \delta} \\ 0 & 1 & 0 \\ -s_{13} e^{-i \delta} & 0 & c_{13}\end{array}\right)$.

Similarly rotation around vector $|1\rangle^{(2)}$ at the angle $\theta_{2}$ is as follows

$$
\begin{aligned}
& |1\rangle^{\operatorname{mix}}=|1\rangle^{(2)}, \\
& |2\rangle^{\operatorname{mix}}=c_{23}|2\rangle^{(2)}+s_{23}|3\rangle^{(2)} \text {, } \\
& |3\rangle^{\operatorname{mix}}=-s_{23}|2\rangle^{(2)}+c_{23}|3\rangle^{(2)}, \\
& \left|v^{\operatorname{mix}}\right\rangle=U^{(3)}|v\rangle^{(2)}, \\
& U^{(3)}=\left(\begin{array}{ccc}
1 & 0 & 0 \\
0 & c_{23} & s_{23} \\
0 & -s_{23} & c_{23}
\end{array}\right) \text {, } \\
& \left|v^{\operatorname{mix}}\right\rangle=U|v\rangle, \quad \text { where } U=U^{(3)} U^{(2)} U^{(1)} \text {, } \\
& U=\left(\begin{array}{ccc}
1 & 0 & 0 \\
0 & c_{23} & s_{23} \\
0 & -s_{23} & c_{23}
\end{array}\right)\left(\begin{array}{ccc}
c_{13} & 0 & s_{13} e^{-i \delta} \\
0 & 1 & 0 \\
s_{13} e^{-i \delta} & 0 & c_{13}
\end{array}\right)\left(\begin{array}{ccc}
c_{12} & s_{12} & 0 \\
-s_{12} & c_{12} & 0 \\
0 & 0 & 1
\end{array}\right) \text {, } \\
& U=\left(\begin{array}{ccc}
c_{13} c_{12} & c_{13} s_{12} & s_{13} e^{-i \delta} \\
-c_{23} s_{12}-c_{12} s_{13} s_{23} e^{i \delta} & c_{12} c_{23}-s_{12} s_{13} s_{23} e^{i \delta} & c_{13} s_{23} \\
s_{23} s_{12}-s_{13} c_{12} c_{23} e^{i \delta} & c_{12} s_{23}-s_{13} c_{23} s_{12} e^{i \delta} & c_{13} c_{23}
\end{array}\right) \text {. }
\end{aligned}
$$

The phase $\delta$ is responsible for the effects of the CP violation which can take values from 0 to $2 \pi$. The mixing angles are parameters for three-neutrino oscillation in vacuum; all real parts of the quadratic products of elements of the mixing matrix entering in the three-neutrino oscillation probabilities are given as $\operatorname{Re}\left(U_{l^{\prime} i} U_{l^{\prime} k}^{*} U_{l i}^{*} U_{l k}\right)$. The individual probability expression for three neutrino flavors changing 
into others can be achieved by solving for individual matrix elements. It is shown below that the probability for neutrino oscillation depend on three mixing angle and two-masssquared difference;

$$
\begin{aligned}
& \operatorname{Re}\left(U_{l^{\prime} i} U_{l^{\prime} k}^{*} U_{l i}^{*} U_{l k}\right) \\
&=\left(U_{22} U_{21}^{*} U_{12}^{*} U_{11}\right), \text { for } l^{\prime}=2, l=1, i=2, k=1 \\
&=\left(c_{23} c_{12}-s_{13} s_{23} s_{12} e^{i \delta}\right)\left(-c_{23} s_{12}-s_{13} s_{23} c_{12} e^{-i \delta}\right) \\
& \times\left(c_{13} s_{12}\right)\left(c_{13} c_{12}\right) \\
&=-\frac{1}{4} c_{13}^{2} \sin 2 \theta_{12} \\
& \times\left[\sin _{2} \theta_{12}\left(c_{23}^{2}-s_{23}^{2} s_{13}^{2}\right)+\cos 2 \theta_{12} \sin 2 \theta_{23} s_{13} \cos \delta_{13}\right], \\
& \operatorname{Re}\left(U_{l^{\prime} i} U_{l^{\prime} k}^{*} U_{l i}^{*} U_{l k}\right) \\
&=\left(U_{23} U_{22}^{*} U_{13}^{*} U_{12}\right) \\
&=\left(c_{13} s_{23}\right)\left(c_{23} c_{12}-s_{13} s_{23} s_{12} e^{-i \delta}\right)\left(s_{13} e^{i \delta}\right)\left(c_{13} s_{12}\right) \\
&=-c_{13}^{2} s_{13} s_{12} s_{23}\left(s_{13} s_{23} s_{12}-c_{23} c_{12} \cos \delta_{13}\right), \\
& \operatorname{Re}\left(U_{l^{\prime} i} U_{l^{\prime} k}^{*} U_{l i}^{*} U_{l k}\right) \\
&=\left(U_{23} U_{21}^{*} U_{13}^{*} U_{11}\right) \\
&=\left(c_{13} s_{23}\right)\left(-c_{23} s_{12}-s_{13} s_{23} c_{12} e^{-i \delta}\right)\left(s_{13} e^{i \delta}\right)\left(c_{13} c_{12}\right) \\
&=-c_{13}^{2} s_{13} c_{12} s_{23}\left(c_{23} s_{12} e^{i \delta}+s_{13} s_{23} c_{12}\right) . \\
&
\end{aligned}
$$

Similarly other expressions can also be derived shown in Table 1.

From the table, it is clear that out of three types of mass mixing, $\Delta m_{21}^{2}, \Delta m_{31}^{2}$, and $\Delta m_{32}^{2}$ only two are independent:

$$
\Delta m_{32}^{2}+\Delta m_{21}^{2}-\Delta m_{31}^{2}=0
$$

Thus neutrino oscillations are only sensitive to the mass squared difference in spite of the actual mass. The solar experiments have inferred the sign of $\Delta m^{2}$ from the MSW effect but the sign of mass squared difference in atmospheric is not known and the condition observed at experiments, $\Delta m_{\text {sol }}^{2} \ll$ $\Delta m_{\text {atmos }}^{2}$, predicts that both kinds of neutrino mixing can occur; one is normal hierarchy $(\mathrm{NH})$ having two light states and one heavier $\left(m_{1} \ll m_{2}<m_{3}\right)$, and the other is inverted hierarchy $(\mathrm{IH}), m_{3}$ is lightest state which assume masses in order $\left(m_{3} \ll m_{1}<m_{2}\right)$ [19]. The two hierarchies are shown in Figure 1.

\section{Atmospheric Neutrino Oscillation Probability}

Atmospheric neutrinos are created by interactions of primary cosmic rays with nuclei in the atmosphere. The neutrinos generate upwardly-going and horizontal muons through decays. The phenomena of atmospheric neutrino oscillations arise from the deficit in upwardly and downward going muon neutrino. Let us calculate the probability for an electron neutrino changing into muon neutrino, as we already discussed that there are three possible cases for electron neutrino changing into muon neutrino, that is, from one mass eigenstate to another:

$$
\begin{aligned}
& P\left(v_{e} \longrightarrow v_{\mu}\right) \\
& =\delta_{12}-4\left[-\frac{1}{4} c_{13}^{2} \sin 2 \theta_{12}\right. \\
& \quad \times\left[\sin 2 \theta_{12}\left(c_{23}^{2}-s_{23}^{2} s_{13}^{2}\right)\right. \\
& \left.\left.\quad+\cos 2 \theta_{12} \sin 2 \theta_{23} s_{13} \cos \delta_{13}\right]\right] \\
& \sin \frac{\Delta m_{21}^{2} L}{2 E}+\delta_{32} \quad \\
& +4\left[-c_{13}^{2} s_{13} s_{12} s_{23}\left(s_{13} s_{23} s_{12}-c_{23} c_{12} \cos \delta_{13}\right)\right] \\
& \quad \times \sin \frac{\Delta m_{32}^{2} L}{4 E} \\
& +\delta_{31}-4\left[-c_{13}^{2} s_{13} c_{12} s_{23}\left(c_{23} s_{12} e^{i \delta}+s_{13} s_{23} c_{12}\right)\right] \\
& \quad \times \sin \frac{\Delta m_{31}^{2} L}{4 E} .
\end{aligned}
$$

For atmospheric neutrinos,

$$
\begin{aligned}
& \Delta m_{32}^{2} \cong \Delta m_{31}^{2} \cong \Delta m_{\mathrm{atm}}^{2}, \\
& \Delta m_{21}^{2} \cong \Delta m_{\mathrm{sol}}^{2} \cong 0 \\
& P\left(v_{e} \longrightarrow v_{\mu}\right)=-4\left(-s_{12}^{2} s_{23}^{2} s_{13}^{2} c_{13}^{2}\right. \\
&+s_{12} s_{23} s_{13} c_{23} c_{12} c_{13}^{2} \cos \delta_{13} \\
&\left.\quad-s_{13}^{2} c_{13}^{2}-s_{12} s_{23} s_{13} c_{23} c_{12} c_{13}^{2} \cos \delta_{13}\right) \\
& \times \sin \frac{\Delta m_{\mathrm{atm}}^{2} L}{4 E} \\
&= \sin ^{2}\left(2 \theta_{13}\right) \sin ^{2}\left(\theta_{23}\right) \sin \frac{\Delta m_{\mathrm{atm}}^{2} L}{4 E}, \\
& P\left(v_{e} \longrightarrow v_{\tau}\right)= \sin ^{2}\left(2 \theta_{13}\right) \cos ^{2}\left(\theta_{23}\right) \sin \frac{\Delta m_{\mathrm{atm}}^{2} L}{4 E} .
\end{aligned}
$$

The survival probability of electron neutrinos is

$$
\begin{aligned}
P\left(v_{e} \longrightarrow v_{e}\right) & =1-\left[P\left(v_{e} \longrightarrow v_{\mu}\right)+P\left(v_{e} \longrightarrow v_{\tau}\right)\right] \\
& =1-\sin ^{2}\left(2 \theta_{13}\right) \sin \frac{\Delta m_{\mathrm{atm}}^{2} L}{4 E} .
\end{aligned}
$$




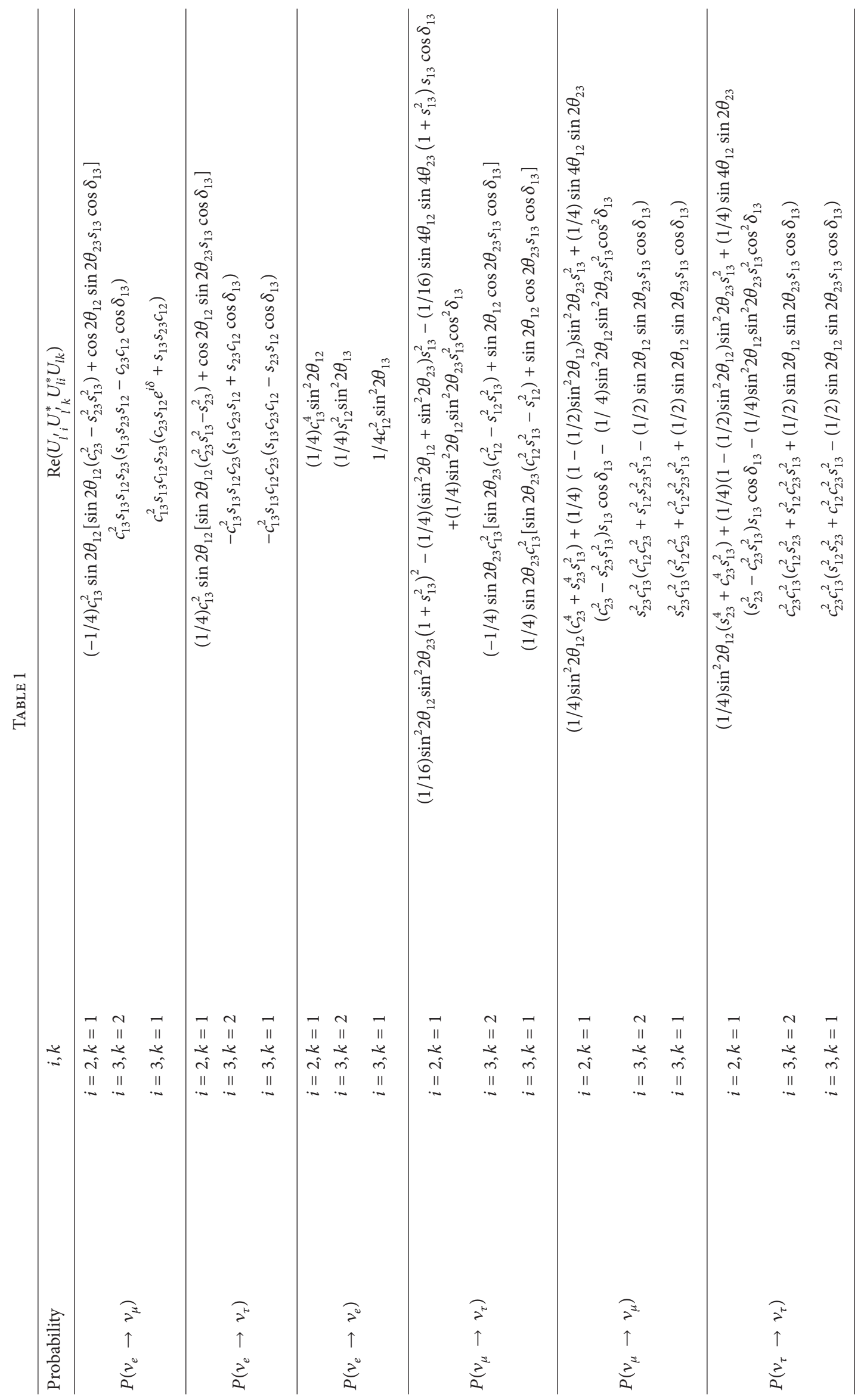


In case of vacuum,

$$
\begin{gathered}
P\left(v_{e} \longrightarrow v_{\mu}\right)=P\left(v_{\mu} \longrightarrow v_{e}\right) \\
P\left(v_{\mu} \longrightarrow v_{e}\right)=\sin ^{2}\left(2 \theta_{13}\right) \sin ^{2}\left(\theta_{23}\right) \sin \frac{\Delta m_{\mathrm{atm}}^{2} L}{4 E} .
\end{gathered}
$$

Similarly we can calculate the probability for muon neutrinos changing into tau neutrinos:

$$
P\left(v_{\mu} \longrightarrow v_{\tau}\right)=\sin ^{2}\left(2 \theta_{23}\right) \cos ^{4}\left(\theta_{13}\right) \sin \frac{\Delta m_{\mathrm{atm}}^{2} L}{4 E}
$$

Now we will calculate the survival probability of muon neutrinos; that is,

$$
\begin{aligned}
P\left(v_{\mu} \longrightarrow v_{\mu}\right)= & 1-\left[P\left(v_{\mu} \longrightarrow v_{e}\right)+P\left(v_{\mu} \longrightarrow v_{\tau}\right)\right] \\
= & 1-\sin ^{2}\left(2 \theta_{13}\right) \sin ^{2}\left(\theta_{23}\right) \\
& +\sin ^{2}\left(2 \theta_{23}\right) \cos ^{4}\left(\theta_{13}\right) \sin \frac{\Delta m_{\mathrm{atm}}^{2} L}{4 E} .
\end{aligned}
$$

These are six probability terms from where we can find the probability for one neutrino changing into another depending upon their flavor.

\section{Solar Neutrino Oscillation Probability}

The difference in the number of solar neutrinos predicted from solar models and the number of neutrinos flowing through earth led to a solar neutrino problem; this created the solar neutrinos as the target for researchers as it can provide a more elaborated picture of stellar evolution and energy resources. The various experiments are focused to measure the solar neutrino flux. Solar neutrino events can also be analyzed by a Monte Carlo simulation study of uncertainties which made use of fluxes from 100 standard solar models [20]. Solar neutrino experimental data constrains that mass squared difference $\Delta m_{21}^{2}$ is only taken where other mass differences are neglected:

$$
\begin{aligned}
& P\left(v_{e} \longrightarrow v_{\mu}\right)=\delta_{12}-4 {\left[-\frac{1}{4} c_{13}^{2} \sin 2 \theta_{12}\right.} \\
& \times {\left[\sin 2 \theta_{12}\left(c_{23}^{2}-s_{23}^{2} s_{13}^{2}\right)\right.} \\
&\left.\left.+\cos 2 \theta_{12} \sin 2 \theta_{23} s_{13} \cos \delta_{13}\right]\right] \\
& \times \sin \frac{\Delta m_{21}^{2} L}{2 E}, \\
& P\left(v_{e} \longrightarrow v_{\mu}\right)=-4\left[-\frac{1}{4} c_{13}^{2} \sin 2 \theta_{12}\right. \\
& \times\left[\sin 2 \theta_{12}\left(c_{23}^{2}-s_{23}^{2} s_{13}^{2}\right)\right. \\
&\left.\left.\quad+\cos 2 \theta_{12} \sin 2 \theta_{23} s_{13} \cos \delta_{13}\right]\right]
\end{aligned}
$$

$$
\begin{aligned}
& \times \sin \frac{\Delta m_{21}^{2} L}{2 E} \\
= & {\left[\sin ^{2} 2 \theta_{12} \cos ^{2}\left(\theta_{13}\right)\right.} \\
& \times\left(\cos ^{2} \theta_{23}-\sin ^{2} \theta_{23} \sin ^{2} \theta_{13}\right) \\
& \left.+\frac{1}{4}\left(\sin 4 \theta_{12} \sin 2 \theta_{13} \cos \theta_{13}\right)\right] \sin \frac{\Delta m_{21}^{2} L}{2 E} .
\end{aligned}
$$

Similarly,

$$
\begin{aligned}
P\left(v_{e} \longrightarrow v_{\tau}\right)=\delta_{12}-4 & {\left[\frac{1}{4} c_{13}^{2} \sin 2 \theta_{12}\right.} \\
& \times\left\{\sin 2 \theta_{12}\left(c_{23}^{2} s_{13}^{2}-s_{23}^{2}\right)+\cos 2 \theta_{12}\right. \\
& \left.\left.\times \sin 2 \theta_{23} s_{13} \cos \delta_{13}\right\}\right] \\
\times & \sin \frac{\Delta m_{21}^{2} L}{4 E} \\
= & -\sin ^{2} 2 \theta_{12} \cos ^{2} \theta_{13} \\
& \times\left(\cos ^{2} \theta_{23} \sin ^{2} \theta_{13}-\sin ^{2} \theta_{23}\right) \\
& -\frac{1}{4} \sin ^{4} \theta_{12} \sin ^{2} \theta_{13}{\left.\sin 2 \theta_{23} \cos \theta_{13}\right]}_{P\left(v_{e} \longrightarrow v_{e}\right)=1-} \\
& \left.\times \sin ^{2} 2 \theta_{12} \cos ^{4} \theta_{13}\right] \sin _{21}^{2} \frac{\Delta m_{21}^{2} L}{2 E}, \\
&
\end{aligned}
$$

\section{Neutrino Oscillations in Matter}

Since neutrinos are weakly interacting, they might interact with matter either through charged-current (CC) or neutralcurrent (NC) interactions [21]. For the charged current interactions, only the electrons participate via $W^{ \pm}$exchange. Mikheev and Smirnov [22] noticed a resonance behavior for specific oscillation and matter density parameters. Therefore the probabilities for neutrino oscillation differ from their vacuum counterparts. Neutral-current flavor interactions can occur for any type of neutrino flavor. Moreover, a neutralcurrent interaction leads to the addition of an extra term in Hamiltonian for flavor oscillation; such a term produces a shift in the eigenvalues, but charged-current interactions give the contribution not only in the form of a change in eigen states but also adds to Hamiltonian an energy proportional to $V=\sqrt{2} G_{F} N_{e}$, where $G_{F}=$ Fermi coupling constant and $N_{e}=$ number of electrons per unit volume. 


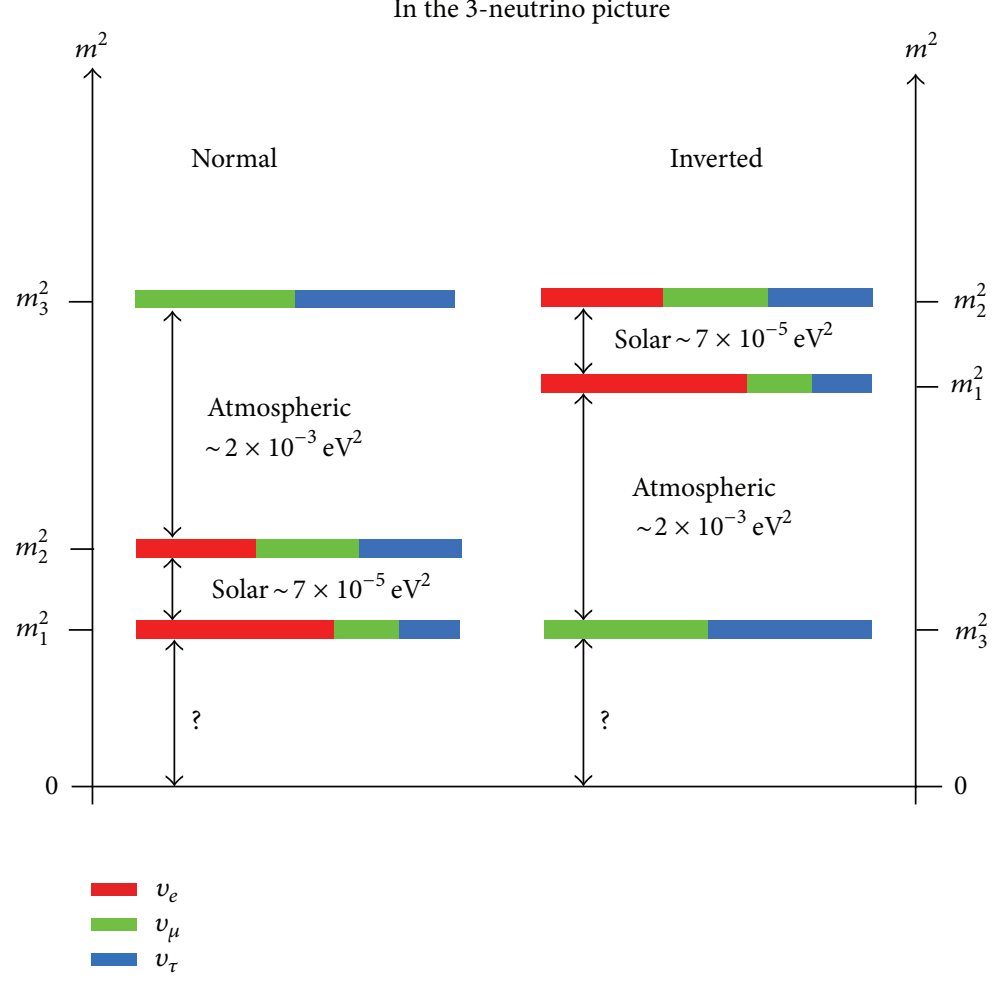

FIgURE 1: Normal and inverted hierarchy.

Neutrinos are produced in flavor eigen states, $\left|v_{\alpha}\right\rangle,(\alpha=$ $e, \mu, \tau)$ created by the interaction of weak gauge bosons with the charged leptons between the source, production point of the neutrinos, and the detector. The state $|\psi(t)\rangle$ of neutrinos with momentum $p$ satisfies the equation

$$
i \frac{\partial}{\partial t}=H_{0}|\psi(t)\rangle
$$

When $H_{0}$ is a free Hamiltonian, the state $|\psi(t)\rangle$ can be expanded over the total system of states of flavor neutrinos $v_{l}$, with momentum $p$,

$$
|\psi(t)\rangle=\sum a_{l}(t)\left|v_{l}\right\rangle
$$

Here

$$
\left|v_{l}\right\rangle=\sum_{i} U_{l i}^{*}\left|v_{i}\right\rangle
$$

$H_{0}\left|v_{i}\right\rangle=E_{i}\left|v_{i}\right\rangle, H_{0}\left|v_{i}\right\rangle=E_{i}\left|v_{i}\right\rangle, E_{i}=\sqrt{p_{i}^{2}+m_{i}^{2}} \simeq p+m_{i}^{2} /$ $2 E$, and $a_{l}(t)=\left\langle v_{l} \mid \psi(t)\right\rangle$ is the amplitude of probability to find $v_{l}$ in state which is described by $|\psi(t)\rangle$.

Therefore,

$$
i \frac{\partial a_{l}^{\prime}(t)}{\partial t}=\sum_{l}\left\langle v_{l}^{\prime}\left|H_{0}\right| a_{l}(t)\right\rangle,
$$

where

$$
\begin{aligned}
& \left\langle v_{l}^{\prime} \mid v_{l}\right\rangle=U_{l^{\prime} l}, \\
& \left\langle v_{l} \mid v_{l^{\prime}}\right\rangle=U_{l^{\prime} l}^{*} .
\end{aligned}
$$

Taking into account this relation, for the free Hamiltonian in the flavor representation we have the following expression:

$$
\left\langle v_{l^{\prime}}\left|H_{0}\right| v_{l}\right\rangle=\sum_{l} U_{l^{\prime} i} E_{i} U_{l i}^{*} \sim p+\sum_{l} U_{l^{\prime} i} \frac{m_{i}^{2}}{2 E} U_{l i}^{*} .
$$

Therefore neutrino evolution equation in the flavor representation is as follows:

$$
i \frac{\partial a(t)}{\partial t}=U \frac{m^{2}}{2 E} U^{\dagger} a(t) .
$$

Let us introduce the function

$$
a^{\prime}(t)=U^{\dagger} a(t)
$$

We find that the function $a^{\prime}(t)$ satisfies the following equation, multiplying by $U^{\dagger}$ on both sides, then

$$
i \frac{\partial}{\partial t} a^{\prime}(t)=\frac{m^{2}}{2 E} a^{\prime}(t) .
$$

It is obvious that the solution of the equation has the form $a^{\prime}(t)=e^{-i\left(m^{2} / 2 E\right)\left(t-t_{0}\right)} a^{\prime}\left(t_{0}\right)$, where, $a^{\prime}\left(t_{0}\right)$ is the wave function at the initial time $t_{0}$.

As the neutrinos propagate in matter, electron neutrino plays a very special role due to coherent forward scattering of neutrinos from electrons so that it leads to an additional contribution in oscillation probability. The forward scattering of electrons of matter with scattering of electrons of matter with neutrinos is called charge current interaction. Therefore 
the probabilities for neutrino oscillation differ from their vacuum counterparts. Earth matter effects for the long baseline have been nicely studied earlier for both hierarchies [23]. Charged current interaction can give contribution only to the process of the elastic scattering of $v_{e}$ on electrons. Moreover, neutral current interactions occur for all flavors, leading to the addition of an extra term in Hamiltonian for the flavor oscillation probability. For the low energy, an effective Hamiltonian of the neutrino interaction was obtained from the diagonal matrix element $\left\langle p\right.$ mat $\left|H_{I}^{\mathrm{CC}}\right| p$ mat $\rangle$, where $H_{I}^{\mathrm{CC}}=\left(G_{F} / \sqrt{2}\right) 2 \overline{\nu_{e L}}(x) \gamma_{\alpha} \nu_{e L} \bar{e}(x) \gamma^{\alpha}\left(1-\gamma_{5}\right) e(x)$ and the vector $\mid p$ mat $\rangle=|p\rangle \mid$ mat $\rangle$. Now substituting the Hamiltonian we obtain $\langle p$ mat $|\left(G_{F} / \sqrt{2}\right) 2 \overline{v_{e L}}(x) \gamma_{\alpha} v_{e L} \bar{e}(x) \gamma^{\alpha}(1-$ $\left.\gamma_{5}\right) e(x) \mid p$ mat $\rangle=\langle p|\langle$ mat $|\left(G_{F} / \sqrt{2}\right) 2 \overline{\nu_{e L}}(x) \gamma_{\alpha} \nu_{e L} \bar{e}(x) \gamma^{\alpha}(1-$ $\left.\gamma_{5}\right) e(x)|p\rangle \mid$ mat $\rangle$.

$\operatorname{But}\left\langle p\left|\left\langle\operatorname{mat}\left|\left(G_{F} / \sqrt{2}\right) 2 \overline{\nu_{e L}}(x) \gamma_{\alpha} v_{e L} \bar{e}(x) \gamma^{\alpha} \gamma_{5} e(x)\right| p\right\rangle\right|\right.$ mat $\rangle=$ 0 as for unpolarized matter $\left\langle\right.$ mat $\left|\bar{e}(x) \gamma^{\alpha} \gamma_{5} e(x)\right|$ mat $\rangle=0$.

Also $\left\langle\operatorname{mat}\left|\bar{e}(x) \gamma^{\alpha} e(x)\right| p\right\rangle \mid$ mat $\rangle=\langle\operatorname{mat}|\bar{e}(x) e(x)| p\rangle \mid$ mat $\rangle$ $\delta_{\alpha 0}=n_{e}(x) \delta_{\alpha 0}$, where $n_{e}(x)$ is the number density at the point $x$. Also, $\left\langle p\left|\overline{v_{e L}}(x) \gamma_{\alpha} v_{e L}\right| p\right\rangle=1$.

Substituting all these values, $H_{I}^{\text {mat }}(t)=\sqrt{2} G_{F} \mathrm{n}_{e}(t) \beta$. Using $\beta_{v_{e}, v_{e}}=1$, all other elements of matrix $\beta$ are equal to zero.

Let us now consider the NC interaction. Induced by the $Z^{0}$ exchange, the Hamiltonian of NC interactions of neutrinos with electrons and nucleons has the form

$$
H_{I}^{\mathrm{CC}}(x)=2 \frac{G_{F}}{\sqrt{2}} \sum_{l=e, \mu, \tau} \bar{v}_{l L}(x) \gamma^{\alpha} v_{l L}(x) j_{\alpha}^{\mathrm{NC}}(x),
$$

where $j_{\alpha}^{\mathrm{NC}}(x)$ is the sum of electron and nucleon (quark) neutral current. For the vector part of the effective hadron neutral current

$$
\begin{gathered}
\nu_{\alpha}^{\mathrm{NC}(N)}(x)=\frac{1}{2} \bar{N}(x) \gamma_{\alpha} \tau_{3} N(x)-2 \sin ^{2} \theta_{w} \bar{p}(x) \gamma_{\alpha} p(x), \\
\text { as } N=\left(\begin{array}{l}
p \\
n
\end{array}\right), \tau_{3}=\left(\begin{array}{cc}
1 & 0 \\
0 & -1
\end{array}\right),
\end{gathered}
$$

where $\theta_{w}$ is the weak angle. Similarly, for the effective part of electron current,

$$
\begin{gathered}
v_{\alpha}^{\mathrm{NC}(n)}(x)=\left(-\frac{1}{2}\right) \bar{n}(x) \gamma_{\alpha} n(x), \\
\nu_{\alpha}^{\mathrm{NC}(e)}(x)=\left(-\frac{1}{2}+2 \sin ^{2} \theta_{w}\right) \bar{e}(x) \gamma_{\alpha} e(x) .
\end{gathered}
$$

For the corresponding matter matrix elements, we have

$$
\begin{gathered}
\left\langle\text { mat }\left|v_{\alpha}^{\mathrm{NC}(e)}(x)\right| \text { mat }\right\rangle=\left(-\frac{1}{2}+2 \sin ^{2} \theta_{w}\right) n_{e}(x) \delta_{\alpha 0}, \\
\left\langle\text { mat }\left|v_{\alpha}^{\mathrm{NC}(p)}(x)\right| \text { mat }\right\rangle=\left(\frac{1}{2}-2 \sin ^{2} \theta_{w}\right) \rho_{p}(x) \delta_{\alpha 0}, \\
\left\langle\text { mat }\left|v_{\alpha}^{\mathrm{NC}(n)}(x)\right| \text { mat }\right\rangle=-\frac{1}{2} \rho_{n}(x) \delta_{\alpha 0} .
\end{gathered}
$$

For the neutral matter, $n_{e}(x)=\rho_{p}(x)$ we conclude that the contributions of electron and proton NC to the effective Hamiltonian cancel each other. Thus

$$
\left\langle\text { mat }\left|j_{\alpha}^{\mathrm{NC}}(x)\right| \text { mat }\right\rangle=-\frac{1}{2} \rho_{n}(x) \delta_{\alpha 0}
$$

By taking into account the effective charged current interaction, only $v_{e}-e$ CC interaction gives a contribution to the effective Hamiltonian. Thus the evolution equation of neutrino has the form

$$
i \frac{\partial a(t)}{\partial t}=\left(U \frac{m^{2}}{2 E} U^{\dagger}+\sqrt{2} G_{F} n_{e}(t) \beta\right) a(t) .
$$

Similarly, for the antineutrinos, effective Hamiltonian differs in sign from the neutrino-electron interactions; thus

$$
\bar{H}_{I}^{\text {mat }}(x)=-\sqrt{2} G_{F} n_{e}(t) \beta \text {. }
$$

Here we are more concerned with the matter effects of a constant density. The total Hamiltonian of neutrino in matter

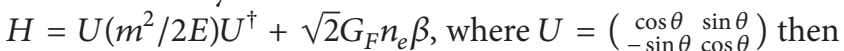
the total effective Hamiltonian is $H=(1 / 2) \operatorname{Tr} H+H^{m}$. Here $(1 / 2) \operatorname{Tr} H=\left(\left(m_{1}^{2}+m_{2}^{2}\right) / 4 E\right)+(1 / 2) \sqrt{2} G_{F} n_{e}$ and $H^{m}$ is the traceless part of Hamiltonian:

$$
\begin{gathered}
H^{m}=\frac{1}{4 E}\left(\begin{array}{cc}
-\Delta m^{2} \cos 2 \theta+A & \Delta m^{2} \sin 2 \theta \\
\Delta m^{2} \sin 2 \theta & \Delta m^{2} \cos 2 \theta-A
\end{array}\right), \\
\text { where } A=2 \sqrt{2} G_{F} n_{e} E \\
H^{m}=U^{m} E^{m} U^{m^{\dagger}}, \\
U^{m}=\left(\begin{array}{cc}
\cos \theta^{m} & \sin \theta^{m} \\
-\sin \theta^{m} & \cos \theta^{m}
\end{array}\right), \\
E^{m}=\left(\begin{array}{cc}
E_{1}^{m} & 0 \\
0 & E_{2}^{m}
\end{array}\right),
\end{gathered}
$$

where $E_{1,2}^{m}= \pm(1 / 4 E) \sqrt{\left(\Delta m^{2} \cos 2 \theta-A\right)^{2}+\left(\Delta m^{2} \sin 2 \theta\right)^{2}}$.

From equation, we find that the mixing angle $\theta^{m}$ is given as:

$$
\begin{aligned}
& \cos 2 \theta^{m}=\frac{\Delta m^{2} \cos 2 \theta-A}{\sqrt{\left(\Delta m^{2} \cos 2 \theta-A\right)^{2}+\left(\Delta m^{2} \sin 2 \theta\right)^{2}}}, \\
& \sin 2 \theta^{m}=\frac{\Delta m^{2} \sin 2 \theta}{\sqrt{\left(\Delta m^{2} \cos 2 \theta-A\right)^{2}+\left(\Delta m^{2} \sin 2 \theta\right)^{2}}} .
\end{aligned}
$$


Three expressions for atmospheric neutrinos for the normal hierarchy are given as follows:

$$
\begin{aligned}
P_{\mu \tau \text { matter }}= & \left(\cos \left[\theta_{13}^{m}\right]\right)^{2} *\left(\sin \left[2 \theta_{23}\right]\right)^{2} \\
& *\left(\sin \left[\frac{\left(1.27 *\left(\Delta m_{31}+A+\Delta m_{31}^{m}\right) * L\right)}{E}\right]\right)^{2} \\
& +\left(\sin \left[\theta_{13}^{m}\right]\right)^{2} *\left(\sin \left[2 \theta_{23}\right]\right)^{2} \\
& *\left(\sin \left[\frac{\left(1.27 *\left(A+\Delta m_{31}-\Delta m_{31}^{m}\right) * L\right)}{E}\right]\right)^{2} \\
& -\left(\cos \left[2 \theta_{23}\right]\right)^{2} *\left(\sin \left[2 \theta_{13}^{m}\right]\right)^{2} \\
& *\left(\sin \left[\theta_{23}\right]\right)^{2} *\left(\sin \left[\frac{\left(1.27 * \Delta m_{31}^{m} * L\right)}{E}\right]\right)^{2}, \\
P_{\mu \mu \text { matter }}= & \left(1-\cos \left[\theta_{13}^{m}\right]\right)^{2} *\left(\sin \left[2 \theta_{23}\right]\right)^{2} \\
& *\left(\sin \left[\frac{\left(1.27 *\left(A+\Delta m_{31}-\Delta m_{31}^{m}\right) * L\right)}{E}\right]\right)^{2}, \\
& *\left(\operatorname { s i n } \left[\frac{\left.\left.\left(1.27 * \Delta m_{31}^{m} * L\right)\right]\right)^{2}}{E}\right.\right. \\
P_{\mu e \text { matter }}= & \left(\sin \left[2 \theta_{13}^{m}\right]\right)^{2} *\left(\sin \left[\theta_{23}\right]\right)^{2} \\
& \left(\frac{{ }^{2}}{E}\right)
\end{aligned}
$$

And for the inverted hierarchy,

$$
\begin{aligned}
& P_{\mu \tau \text { matter }}=\left(\cos \left[\theta_{13}^{m}\right]\right)^{2} *\left(\sin \left[2 \theta_{23}\right]\right)^{2} \\
& *\left(\sin \left[\frac{\left(1.27 *\left(A-\Delta m_{31}+\Delta m_{31}^{m}\right) * L\right)}{E}\right]\right)^{2} \\
& +\left(\sin \left[\theta_{13}{ }^{m}\right]\right)^{2} *\left(\sin \left[2 \theta_{23}\right]\right)^{2} \\
& *\left(\sin \left[\frac{\left(1.27 *\left(A+\Delta m_{31}-\Delta m_{31}^{m}\right) * L\right)}{E}\right]\right)^{2} \\
& -\left(\cos \left[2 \theta_{23}\right]\right)^{2} *\left(\sin \left[2 \theta_{13}^{m}\right]\right)^{2} \\
& *\left(\sin \left[\theta_{23}\right]\right)^{2} *\left(\sin \left[\frac{\left(1.27 * \Delta m_{31}^{m} * L\right)}{E}\right]\right)^{2}, \\
& P_{\mu \mu \mathrm{matter}}=\left(1-\cos \left[\theta_{13}^{m}\right]\right)^{2} *\left(\sin \left[2 \theta_{23}\right]\right)^{2} \\
& *\left(\sin \left[\frac{\left(1.27 *\left(A-\Delta m_{31}-\Delta m_{31}^{m}\right) * L\right)}{E}\right]\right)^{2}, \\
& P_{\mu e m a t t e r}=\left(\sin \left[2 \theta_{13}^{m}\right]\right)^{2} *\left(\sin \left[\theta_{23}\right]\right)^{2} \\
& *\left(\sin \left[\frac{\left(1.27 * \Delta m_{31}^{m} * L\right)}{E}\right]\right)^{2} \text {. }
\end{aligned}
$$

\section{Oscillation Parameters and Masses}

Neutrino oscillation is new physics beyond the standard model and requires the addition of a new field and new parameters to the standard model. Although atmospheric and solar neutrinos are having different origin, yet they exhibit the same phenomenon of oscillations among their flavors. The parameters involved in three-flavor oscillations can be estimated from the neutrinos coming from the sun and atmospheric and nuclear reactors and accelerators. Solar experiments detect neutrinos generated in the core of the sun due to thermonuclear reactions and must have the energy of the order of $0.2-15 \mathrm{MeV}$, whereas atmospheric experiments detect the neutrino produced in a cascade initiated by Cosmic rays collisions with nuclei in the Earth's atmosphere and has a source detector at a distance of several tons of meters with a range $L / E<1 \mathrm{~m} / \mathrm{MeV}$. Oscillation parameters historically fall into four categories in mixing angle-mass splitting parameter space: vacuum oscillations (VAC), "LOW", small mixing angle (SMA), and large mixing angle (LMA). Similarly based on the distance from source to detector, detectors can be SBL, LBL, VSBL, and VLBL. A global analysis of different experiments can provide best fit values of different parameters. The two large mixing angles $\theta_{12}$ and $\theta_{23}$ have been found to be of the order of $\sim 34^{\circ}$ and $\sim 45^{\circ}$, respectively, but the third mixing angle $\theta_{13}$ is suppressed by different experiments. Recent searches for $\theta_{13}$, however, oppose suppression of this mixing angle. Moreover sign of $\Delta m_{31}^{2}$ is still unknown; therefore two types of neutrino spectrum are possible, one is normal hierarchy $\left(m_{1} \ll m_{2}<\right.$ $m_{3}$ ) whereas the other is inverted hierarchy which assume masses in order $\left(m_{3} \ll m_{1}<m_{2}\right)$. Three mixing angles and the mass differences from global analysis of data [24] are given below:

$$
\begin{aligned}
\Delta m_{21}^{2} & =7.59 \pm 0.20_{-0.69}^{+0.61} \times 10^{-5} \mathrm{eV}^{2} \\
\Delta m_{31}^{2} & =+2.46 \pm 0.12( \pm 0.37) \times 10^{-3} \mathrm{eV}^{2} \\
& =-2.36 \pm 0.11 \pm 0.37 \mathrm{eV}^{2} \\
\theta_{23} & =42.8_{-2.9}^{+4.7}\left(\begin{array}{l}
+10.7 \\
-7.3
\end{array}\right) \\
\theta_{12} & =34.4 \pm 1.0\left(\begin{array}{l}
+3.2 \\
-2.9
\end{array}\right) \\
\theta_{13} & =5.6_{-2.7}^{+3.0}
\end{aligned}
$$

The parameter affecting the neutrino oscillation in matter is given by $A=2 \sqrt{2} G_{F} n_{e} E$ where $G_{F}$ is Fermi constant, $n_{e}$ is the electron number density, and $E$ is the energy of neutrino beam. The parameter $A$ is almost constant for the long baseline experiment with high precision as the beam does not penetrate deeply into the earth without affecting the different layers of the earth, but in sun, density decreases exponentially from center to core of earth which further leads to resonance enhancement of oscillations known as the MSW effect [22]. We here restrict ourselves to the matter effects with constant density for atmospheric neutrinos only. The graph for probability versus energy at three different lengths in vacuum as well as in matter (for normal and inverted 


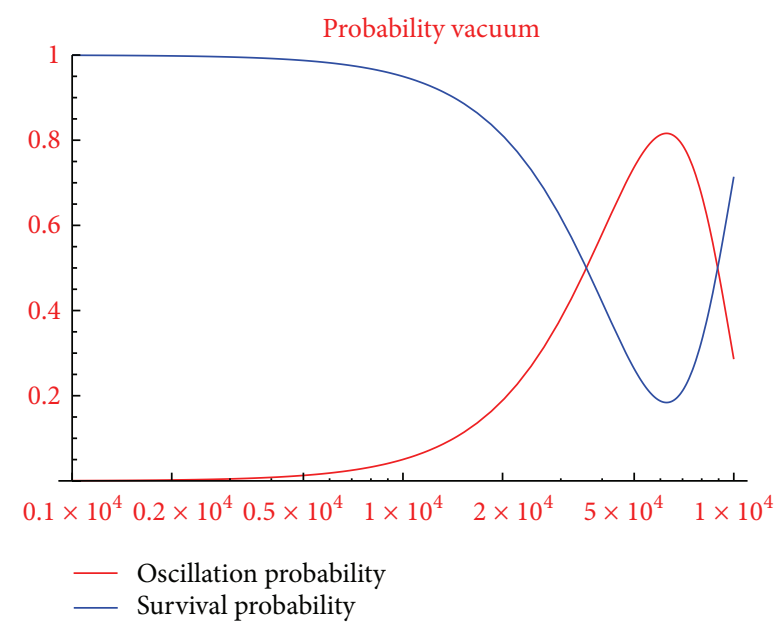

Figure 2: Oscillations and survival probabilities.

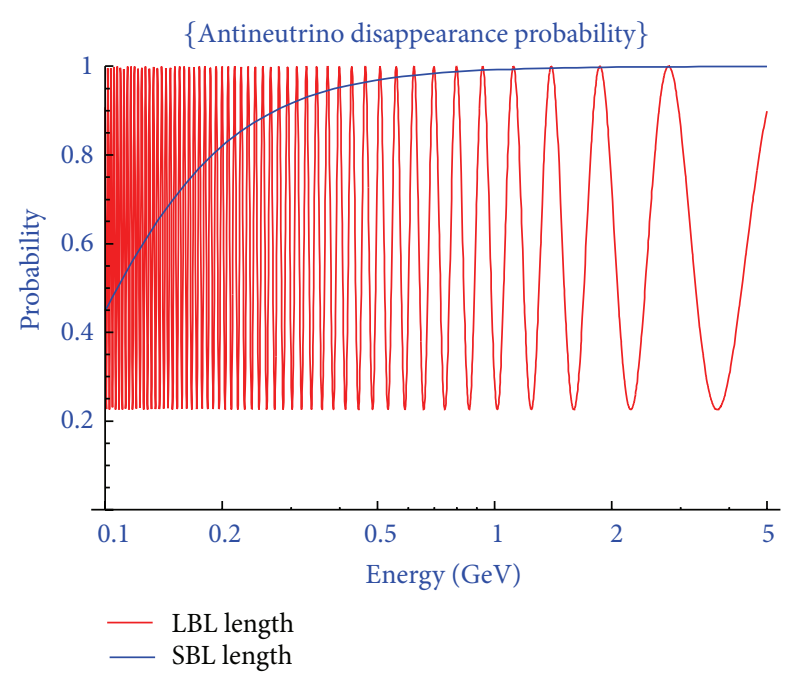

FIGURE 3

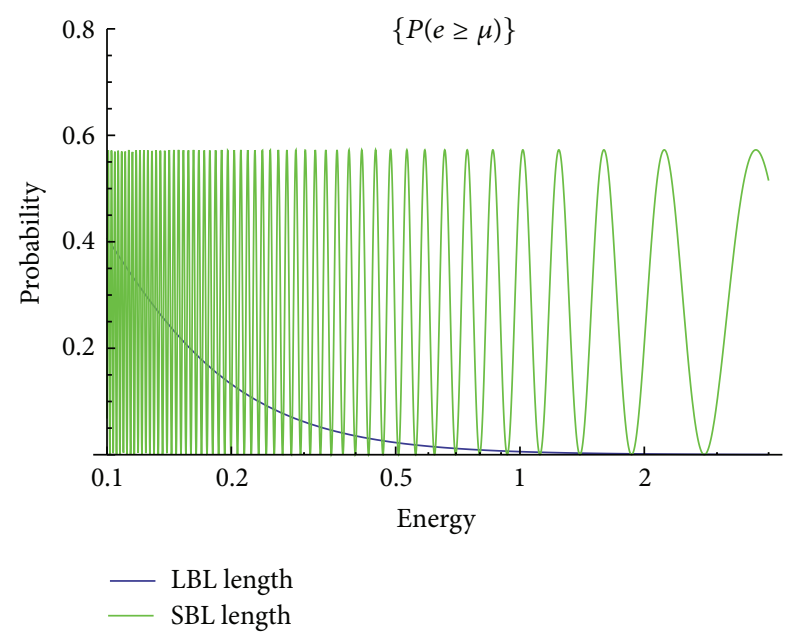

FIGURE 4

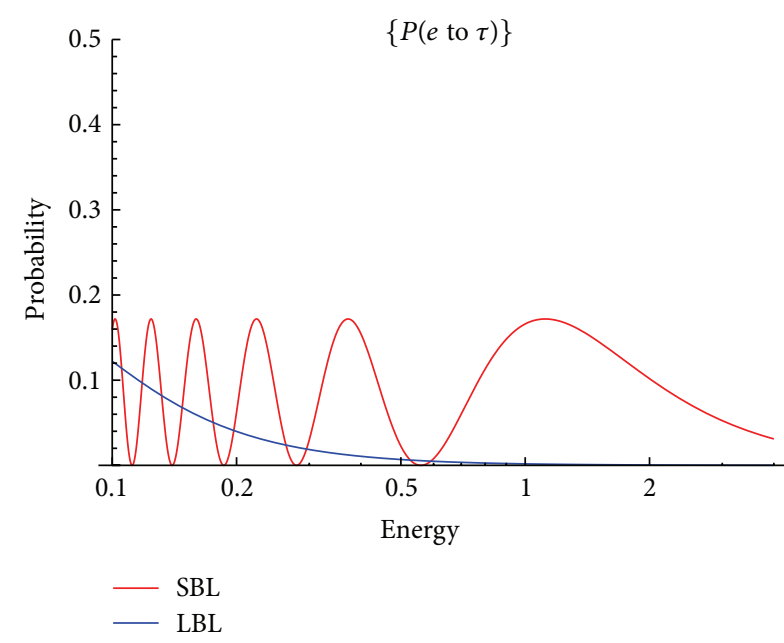

Figure 5

hierarchy) for $v_{\mu}$ to $v_{e}, v_{\mu}$ to $v_{\tau}$, and $v_{\mu}$ to $v_{\mu}$ shows that the presence of matter affects the neutrino oscillation probability the most in case of the $v_{\mu}$ to $v_{e}$ oscillations. This may be due to the reason that the charged current interaction with the electrons of the medium directly affects the oscillation probability in case of electrons only. This proves the fact that the matter effects are flavor dependent. Moreover, oscillations become more frequent at low energy and the increase in length results in increase of the energy range which is more sensitive to oscillations but affects to a very lesser extent of magnitude of transition probability. Oscillation probability is the more of magnitude in case of $\mu$ to $\tau$ rather than an electron which concludes that a mass eigen state is more composed of $\mu$ and $\tau$ rather than an electron for atmospheric neutrinos especially. The smallness of $v_{e}$ part of total mass eigen state is measured by the mixing angle $\theta_{13}$. For solar neutrino oscillation probability, reactor-based neutrino experiments and detectors are designed as different experiments are designed in order to be sensitive to different values of $\Delta m^{2}$, by choosing the appropriate value of $L / E$, the neutrino beam consisting of $v_{e}$ when coming out of the source and having energy only $\sim 1 \mathrm{MeV}$ that is, in order to satisfy $\Delta m^{2} L / 2 E \sim 1$ and $L$ can be of order such that $L / E<=1 \mathrm{~km} / \mathrm{MeV}$. Solar neutrino experiments have sensitivity for $\Delta m^{2}$ of very small value. SNO has been extremely successful so far to prove neutrino flavor change because it was designed in such a way to detect the changed flavor of solar neutrinos, that is, the flavor in which $v_{e}$ changed. After analyzing the results from flux ratios, it was found that day-night asymmetry has more sensitivity to the value of $\Delta m_{12}^{2}$. The results favored large mixing angle solutions. The $\mathrm{CHOOZ}$ [25] experiment put forward that the electron antineutrino survival probability and its oscillation probability from electron to muon and tau are excluded for $\Delta m^{2} \geq 8 \times 10^{-4} \mathrm{eV}^{2}$ and at maximum mixing $\sin ^{2} 2 \theta \geq 0.17$. The chain of experiment continued with the KamLAND [8] experiment which provided evidence of antineutrino disappearance probability for the very first time with a long baseline length of the order of $\sim 175 \mathrm{~km}$ as shown in Figure 6. All reactor-based neutrinos consider only 


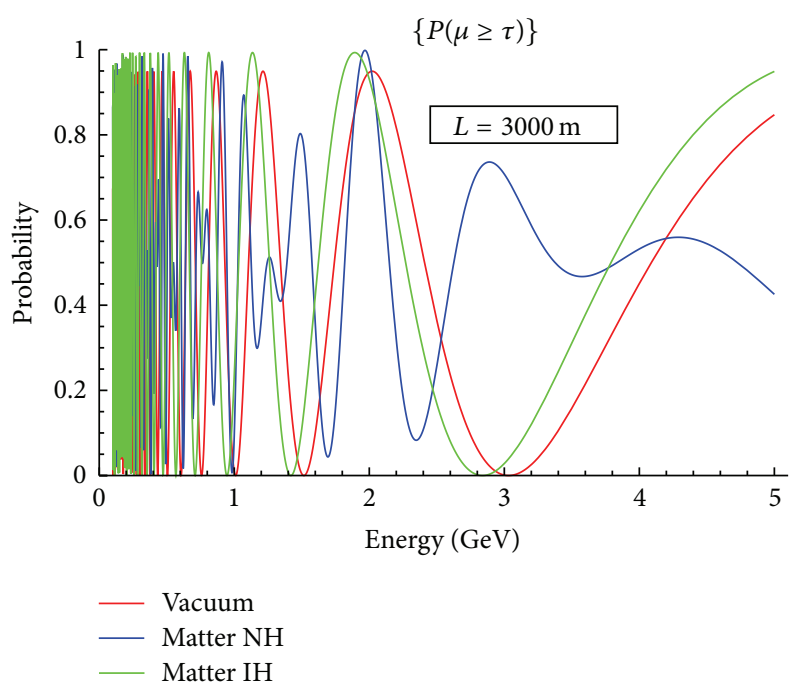

(a)

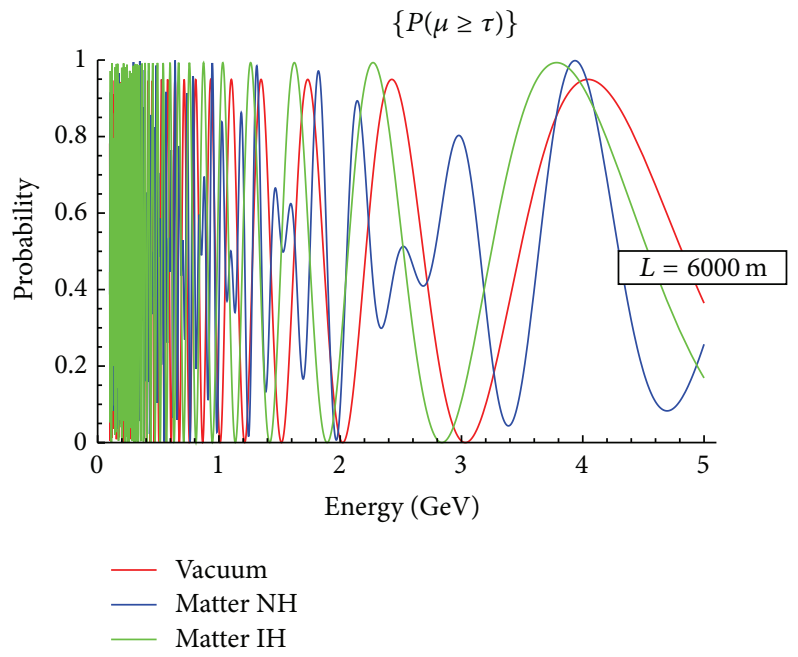

(b)

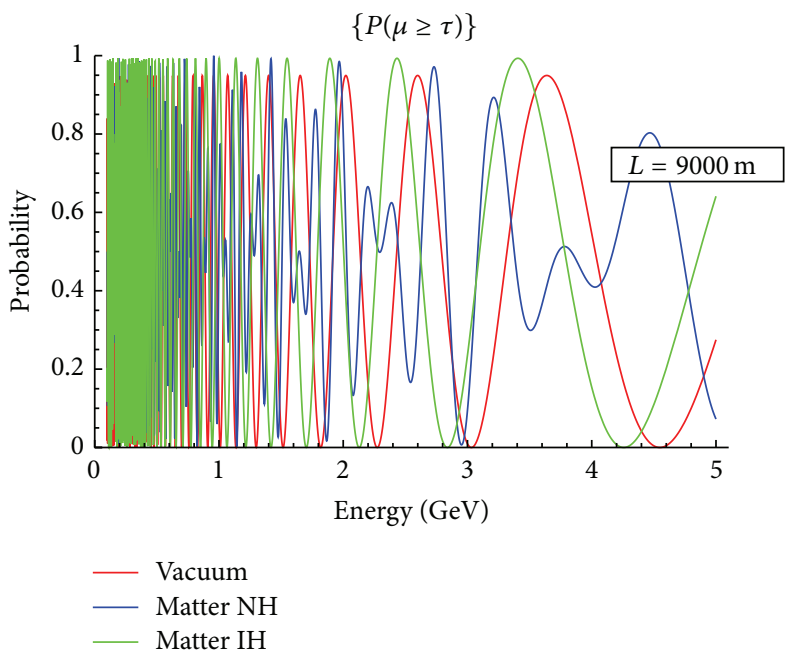

(c)

FiguRe 6

the vacuum part of the oscillation probability as matter effects are negligible at low energy. Transitions from electron to $\mu$ 's and $\tau$ 's are shown in Figures 7 and 8. Two plots here show that the maximum oscillations are from $e$ to $\mu$ for solar neutrinos.

\section{Summary and Conclusion}

In summary, two flavor oscillations are discussed here for the probability measurements and moreover generalized to three flavor despite in vacuum and matter. In the era of neutrino physics, vast amount of the information for oscillation parameters we have in hand, still the situation is not clear yet. Neutrino oscillations helped to solve the greatest mystery in solar world but actual mass of the neutrinos is still unknown. The recent searches for neutrinos are more focused on the estimation of parameter $\theta_{13}$ [25] and the speed of neutrinos. Some more pieces of information on the mass hierarchy and $\mathrm{CP}$ violating phase can help us to get more detailed information on oscillation phenomenology. Future experiments are more concerned to find the accurate and precise measurement of $\theta_{13}$. The accurate determination of $\theta_{13}$ can provide the more elaborated information on $\mathrm{CP}$ violating phase $\delta$ and mass hierarchy. It is proposed that a sign of $\Delta m_{31}^{2}$ can also be well determined. Reactor experiments are unique in the sense that they can give a value of $\theta_{13}$ up to a higher precision value. That's why more recent experiments like Double CHOOZ, T2K, Nova, Daya Bay, and MINOS are designed in such a way that they are expected to measure this angle. Nuclear reactor experiments are more efficient in determination of $\theta_{13}$ as for the case of long baseline experiments sensitivity is reduced due to effect of transition parameters and matter effects. Nuclear reactors are 


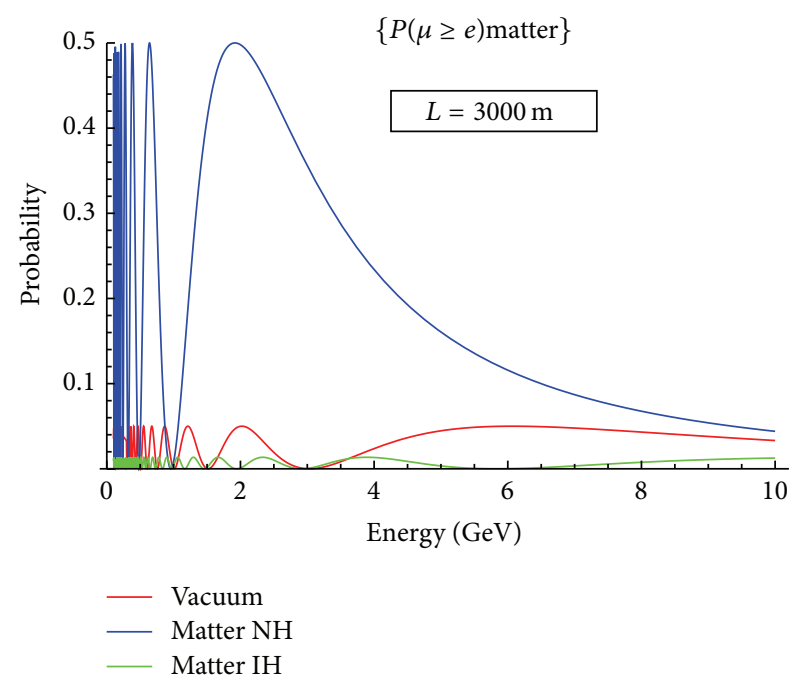

(a)

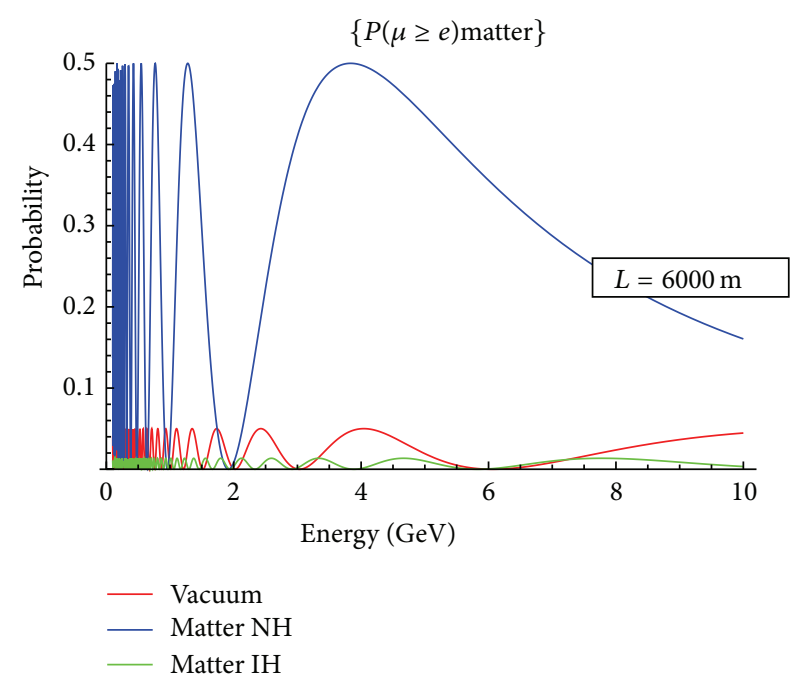

(b)

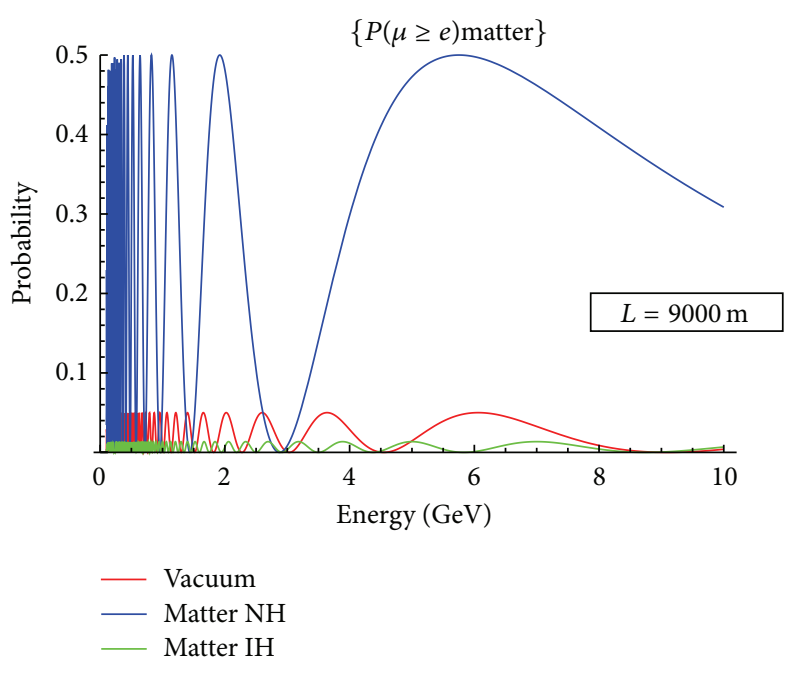

(c)

Figure 7

very intense sources of $\bar{v}_{e}$ 's coming from the $\beta$-decay of the neutron-rich fission fragments. If relaxation $\theta_{13}$ is avoided, that antineutrino disappearance probability becomes

$$
\begin{aligned}
P\left(\bar{v}_{\mathrm{e}} \longrightarrow \bar{v}_{\mathrm{e}}\right)=1 & -\sin _{2 \theta_{13}}^{2} \sin ^{2} \Delta m_{31}^{2} \frac{L}{2 E} \\
& -\cos 4_{\theta_{13}} \sin _{\theta_{12}}^{2} \sin ^{2} \Delta m_{31}^{2} \frac{L}{2 E} .
\end{aligned}
$$

Thus sensitivity of $\theta_{13}$ can be seen more at small energy and short baseline rather than long baseline as shown in Figure 9. The present information about this angle can be determined through global analysis of data. Gonzalez-Garcia et al. predicted the following limits on $\theta_{13}$ from various experiments [26]:

$$
\sin _{\theta_{13}}^{2}<\left\{\begin{array}{c}
0.060(0.089)(\text { solar }+ \text { KAMLAND }) \\
0.027(0.058)(\mathrm{CHOOZ}+\text { atm }+ \text { K2K+MINOS }) \\
0.035(0.056)(\text { Global data })
\end{array}\right\} .
$$

But what would happen if this angle is large? Earlier experiments assumed $\theta_{13}$ as zero because of some discrete symmetries present in the mixing parameters. Now the question arises how these symmetries are changed if $\theta_{13}$ is large or the new parameter ranges are compatible with the PMNS matrix $U$ and whether the more resolved values of two mass differences is expected or not? What are the changes expected in the neutrino beam energy to measure more accurately the mixing parameters? 

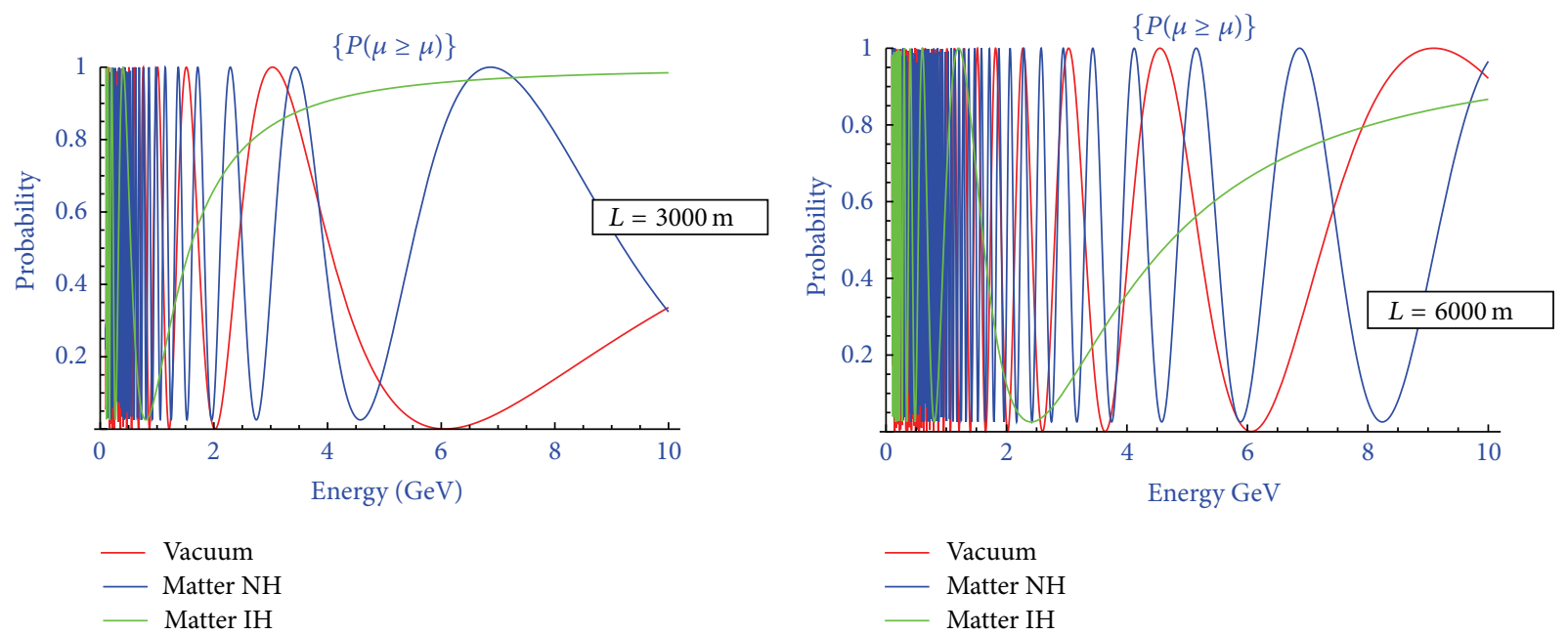

(a)

(b)

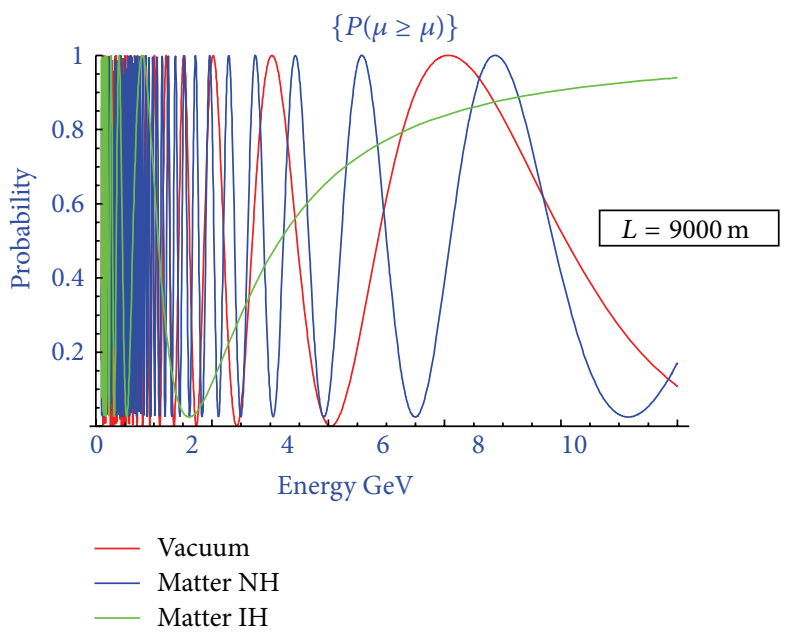

(c)

Figure 8

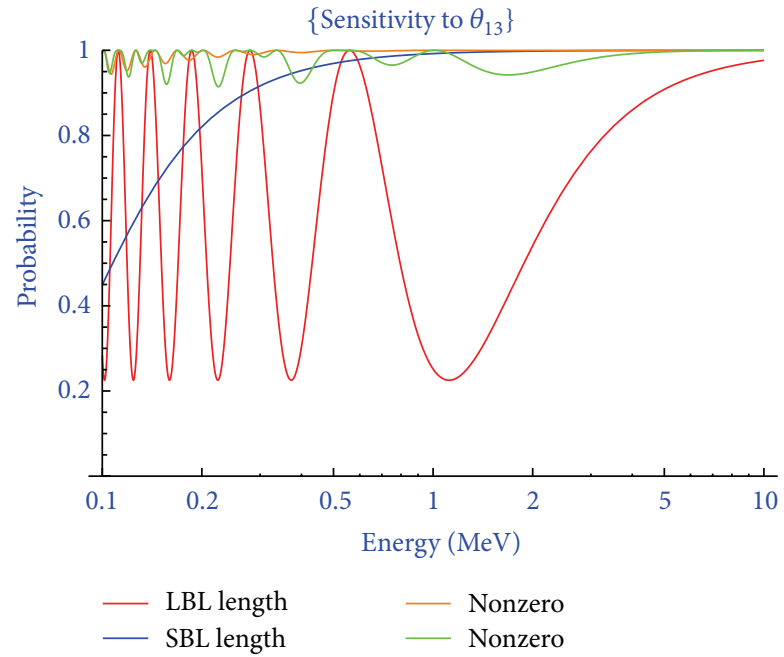

Figure 9 


\section{Appendix}

\section{Oscillation Plots for Vacuum and Matter}

For more details, see Figures 2, 3, 4, 5, 6, 7, 8, and 9 and Table 1.

\section{References}

[1] V. Gribov and B. Pontecorvo, "Neutrino astronomy and lepton charge," Physics Letters B, vol. 28, no. 7, pp. 493-496, 1969.

[2] B. T. Cleveland, T. Daily, R. Davis Jr. et al., "Measurement of the solar electron neutrino flux with the homestake chlorine detector," The Astrophysical Journal, vol. 496, p. 505, 1998.

[3] J. N. Bahcall, A. M. Serenelli, and S. Basu, "10,000 Standard solar models: a monte carlo simulation," Astrophysical Journal, Supplement Series, vol. 165, no. 1, pp. 400-431, 2006.

[4] M. Cribier, "Results of the whole GALLEX experiment," Nuclear Physics B Proceedings of science, vol. 70, no. 1-3, pp. 284-291, 1999.

[5] J. N. Abdurashitov, "Solar neutrino flux measurements by the Soviet-American gallium experiment (SAGE) for half the 22-year solar cycle," Journal of Experimental and Theoretical Physics, vol. 95, pp. 181-193, 2002, Zhurnal Eksperimentalnoi i Teoreticheskoi Fiziki, vol. 122, pp. 211-226, 2002.

[6] S. N. Ahmed, A. E. Anthony, E. W. Beier et al., "Measurement of the total active ${ }^{8} \mathrm{~B}$ solar neutrino flux at the Sudbury Neutrino Observatory with enhanced neutral current sensitivity," Physical Review Letters, vol. 92, no. 18, Article ID 181301, 6 pages, 2004.

[7] Y. Fukuda, T. Hayakawa, E. Ichihara et al., "Evidence for oscillation of atmospheric neutrinos," Physical Review Letters, vol. 81, no. 8, pp. 1562-1567, 1998.

[8] S. Abe, T. Ebihara, S. Enomoto et al., "Precision measurement of neutrino oscillation parameters with KamLAND," Physical Review Letters, vol. 100, no. 22, Article ID 221803, 2008.

[9] K. Abe, N. Abgrall, Y. Ajima et al., "Indication of electron neutrino appearance from an accelerator-produced off-axis muon neutrino beam," Physical Review Letters, vol. 107, no. 4, Article ID 041801, 2011.

[10] P. Adamson, C. Andreopoulos, K. E. Arms et al., "Study of muon neutrino disappearance using the Fermilab Main Injector neutrino beam," Physical Review D, vol. 77, no. 7, Article ID 072002, 2008.

[11] W. W. M. Allison, G. J. Alner, D. S. Ayres et al., "Neutrino oscillation effects in Soudan 2 upward-stopping muons," Physical Review D, vol. 72, no. 5, Article ID 052005, 2005.

[12] E. Aliu, S. Andringa, S. Aoki et al., "Evidence for muon neutrino oscillation in an accelerator-based experiment," Physical Review Letters, vol. 94, no. 8, Article ID 081802, 2005.

[13] J. Hosaka, K. Ishihara, J. Kameda et al., "Solar neutrino measurements in Super-Kamiokande-I," Physical Review D, vol. 73, no. 11, Article ID 112001, 2006.

[14] J. P. Cravens, K. Abe, T. Iida et al., "Solar neutrino measurements in Super-Kamiokande-II," Physical Review D, vol. 78, no. 3, Article ID 032002, 2008.

[15] R. Wendell, C. Ishihara, K. Abe et al., "Atmospheric neutrino oscillation analysis with subleading effects in SuperKamiokande I, II, and III," Physical Review D, vol. 81, no. 9, Article ID 092004, 2010.

[16] M. H. Ahn, S. Aoki, Y. Ashie et al., "Search for electron neutrino appearance in a $250 \mathrm{~km}$ long-baseline experiment," Physical
Review Letters, vol. 93, no. 5, Article ID 051801, pp. 051801-1, 2004.

[17] P. Adamson, C. Andreopoulos, R. Armstrong et al., "Measurement of the neutrino mass splitting and flavor mixing by minos," Physical Review Letters, vol. 106, no. 18, Article ID 181801, 2011.

[18] B. Kayser, "On the quantum mechanics of neutrino oscillation," Physical Review D, vol. 24, p. 110, 1981.

[19] C. Giunti, C. W. Kim, J. A. Lee, and U. W. Lee, "Treatment of neutrino oscillations without resort to weak eigenstates," Physical Review D, vol. 48, no. 9, pp. 4310-4317, 1993.

[20] H. Nunokawa, S. Parke, and J. W. F. Valle, "CP violation and neutrino oscillations," Progress in Particle and Nuclear Physics, vol. 60, no. 2, pp. 338-402, 2008.

[21] J. N. Bahcall, A. M. Serenelli, and S. Basu, "New solar opacities, abundances, helioseismology, and neutrino fluxes," Astrophysical Journal, vol. 621, no. 1, pp. L85-L88, 2005.

[22] L. Wolfenstein, "Neutrino oscillations in matter," Physical Review D, vol. 17, no. 9, pp. 2369-2374, 1978.

[23] R. Gandhi, P. Ghoshal, S. Goswami, P. Mehta, and S. U. Sankar, "Earth matter effects at very long baselines and the neutrino mass hierarchy," Physical Review D, vol. 73, no. 5, Article ID 053001, 2006.

[24] S. P. Mikheev and A. Y. Smirnov, "Resonance amplification of oscillations in matter and spectroscopy of solar neutrinos," Soviet Journal of Nuclear Physics, vol. 42, pp. 913-917, 1985.

[25] W. Winter, "Minimal neutrino beta beam for large $\theta_{13}$," Physical Review D, vol. 78, no. 3, Article ID 037101, 2008.

[26] M. C. Gonzalez-Garcia, M. Maltoni, and J. Salvado, "Direct determination of the solar neutrino fluxes from solar neutrino data," Journal of High Energy Physics, vol. 1004, no. 5, article 072, p. $56,2010$. 

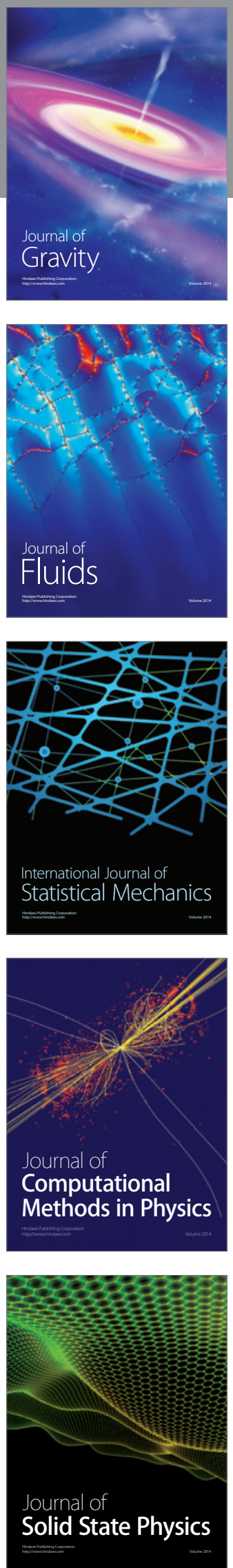

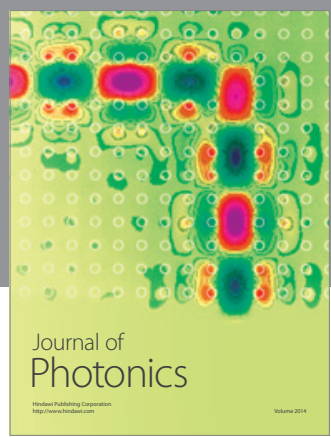

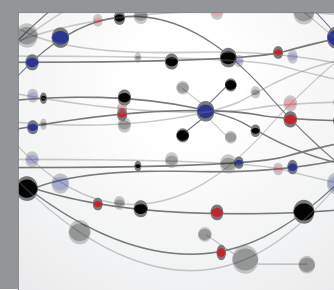

The Scientific World Journal

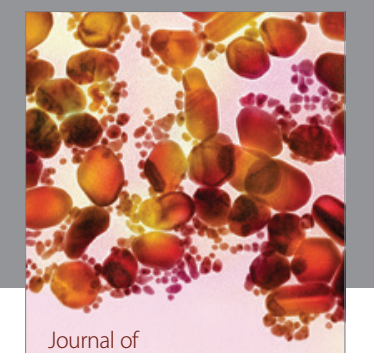

Soft Matter
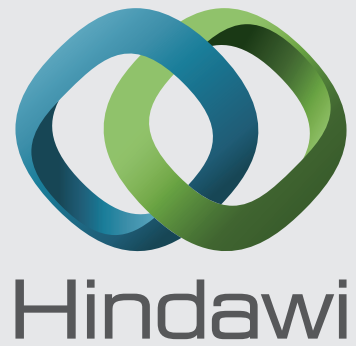

Submit your manuscripts at

http://www.hindawi.com
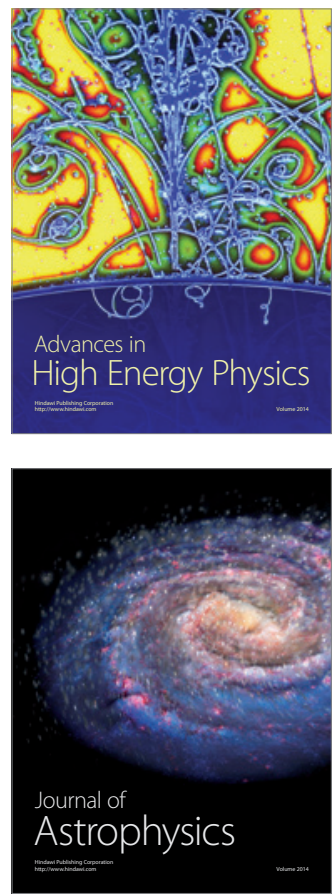
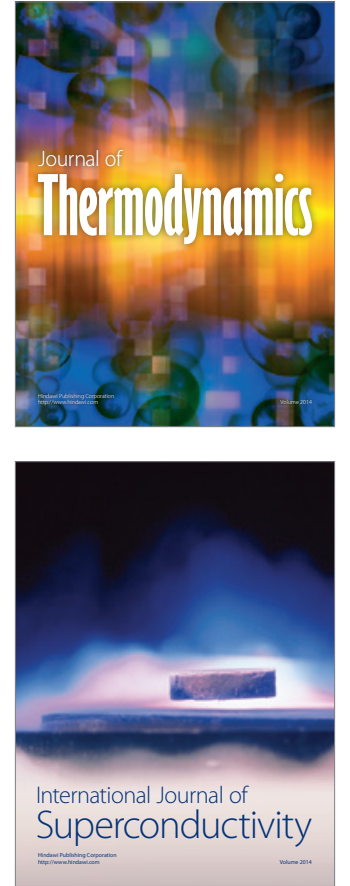
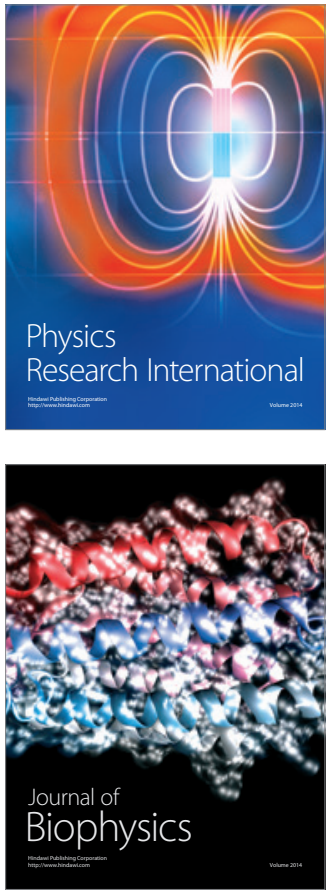
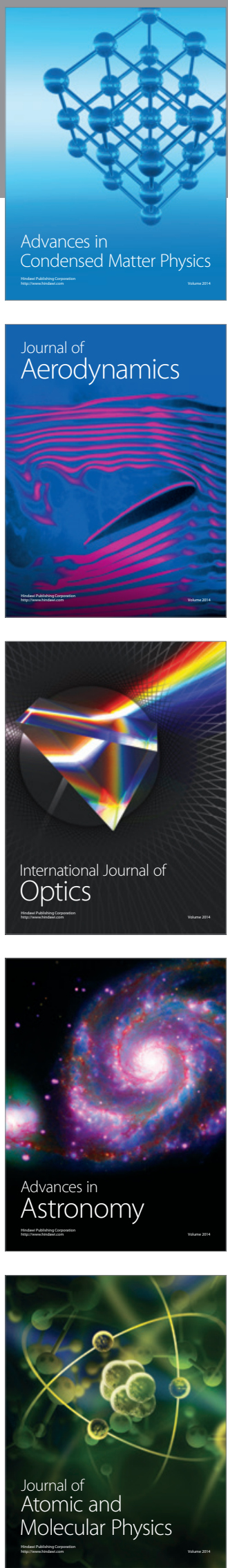